\title{
Automatic segmentation of the lumen region in intravascular images of the coronary artery
}

\author{
Danilo Samuel Jodas ${ }^{\mathrm{a}, \mathrm{c}}$, Aledir Silveira Pereira ${ }^{\mathrm{b}}$, João Manuel R.S. Tavares ${ }^{\mathrm{c}, *}$ \\ ${ }^{a}$ CAPES Foundation, Ministry of Education of Brazil, Brasília - DF, 70040-020, Brazil \\ ${ }^{\mathrm{b}}$ Universidade Estadual Paulista "Júlio de Mesquita Filho", Rua Cristóvão Colombo, 2265, 15054-000, S. J. do Rio Preto, Brazil \\ ${ }^{\mathrm{c}}$ Instituto de Ciência e Inovação em Engenharia Mecânica e Engenharia Industrial, Faculdade de Engenharia, Universidade do Porto, Rua Dr. Roberto Frias, \\ $s / n, 4200-465$, Porto, Portugal
}

\section{A R T I C L E I N F O}

\section{Article history:}

Received 29 December 2016

Revised 3 June 2017

Accepted 9 June 2017

Available online 10 June 2017

\section{Keywords:}

Medical imaging

Intravascular ultrasound

Image pre-processing

Image segmentation

\begin{abstract}
A B S T R A C T
Image assessment of the arterial system plays an important role in the diagnosis of cardiovascular diseases. The segmentation of the lumen and media-adventitia in intravascular (IVUS) images of the coronary artery is the first step towards the evaluation of the morphology of the vessel under analysis and the identification of possible atherosclerotic lesions. In this study, a fully automatic method for the segmentation of the lumen in IVUS images of the coronary artery is presented. The proposed method relies on the $\mathrm{K}$-means algorithm and the mean roundness to identify the region corresponding to the potential lumen. An approach to identify and eliminate side branches on bifurcations is also proposed to delimit the area with the potential lumen regions. Additionally, an active contour model is applied to refine the contour of the lumen region. In order to evaluate the segmentation accuracy, the results of the proposed method were compared against manual delineations made by two experts in 326 IVUS images of the coronary artery. The average values of the Jaccard measure, Hausdorff distance, percentage of area difference and Dice coefficient were $0.88 \pm 0.06,0.29 \pm 0.17 \mathrm{~mm}, 0.09 \pm 0.07$ and $0.94 \pm 0.04$, respectively, in 324 IVUS images successfully segmented. Additionally, a comparison with the studies found in the literature showed that the proposed method is slight better than the majority of the related methods that have been proposed. Hence, the new automatic segmentation method is shown to be effective in detecting the lumen in IVUS images without using complex solutions and user interaction.
\end{abstract}

(C) 2017 Elsevier B.V. All rights reserved.

\section{Introduction}

According to the World Health Organization (WHO), coronary artery diseases were responsible for the death of 7.3 million people around the world in 2008 (Mendis et al., 2011). Atherosclerosis is an underlying disease responsible for the occurrence of heart attacks and strokes. Atherosclerotic plaques are formed when fatty material and cholesterol are deposited inside the lumen of the artery, reducing the blood flow through the vessel and increasing the risk of blood clots that can cause heart attacks and strokes. Thus, in order to prevent such risks a treatment plan or even stenting procedures should be established based on image-based technologies.

\footnotetext{
* Corresponding author.

E-mail addresses: danilojodas@gmail.com (D.S. Jodas), aledir@sjrp.unesp.br (A.S. Pereira), tavares@fe.up.pt (J.M.R.S. Tavares).
}

URL: http://www.fe.up.pt/ tavares (J.M.R.S. Tavares)
Technological advances in computerized systems for imagingbased diagnosis are able to detect and analyse cardiovascular diseases. Intravascular Ultrasound (IVUS) is an imaging procedure that allows the evaluation of the arterial morphology by means of the introduction of a catheter equipped with an ultrasound transducer inside the vessel to be studied. The catheter slides through a guidewire placed in the blood vessel near the segment of interest in order to acquired images of the affected region. Images acquired from IVUS imaging systems allow experts to envisage atherosclerotic lesions and the shape and size of the vessels under analysis.

The segmentation of IVUS images plays an important role in evaluating the morphology of the vessel under study and obtaining important information such as the area and diameter of the lumen, the presence and volume of atherosclerotic plaques and the identification of the atherosclerotic plaque components (Nair et al., 2002; Diethrich et al., 2006; König and Klauss, 2007; Broersen et al., 2016). Since a large number of image frames are acquired from a single IVUS exam, the manual identification of the structures of interest becomes a time-consuming and 
laborious task. Therefore, the automatic segmentation of IVUS images is demanded for expediting the assessment of the morphology of the vessel and the treatment planning of atherosclerotic lesions.

The segmentation of the lumen and media-adventitia regions in IVUS images has been an intensive focus of research (Katouzian et al., 2012). The presence of calcifications, shadows, transducer reflection, speckle noises and bifurcations, as well as the variations of the grayscale intensities inside the same structure, represent a challenge to the development of a fully automatic segmentation method. The presence of the first two artefacts, i.e. calcifications and shadows, is an obstacle to the efficient segmentation of the media-adventitia region, whereas the transducer reflection and speckle noises represent a challenge to identify the region corresponding to the lumen successfully. Additionally, the segmentation of the lumen in bifurcation regions represents a challenge due to the extension of the low-intensity values from the lumen region to the borders of the IVUS image.

This article proposes a method to automatically detect the lumen boundary in IVUS images of coronary arteries. An initial version of this method was applied in the segmentation of the lumen in magnetic resonance (MR) images of carotid arteries (Jodas et al., 2016). The approach was based on the assumption that the lumen is a low-intensity region with an approximately circular shape. Thus, a circularity index, combined with a centre index and the number of regions at the border of the image were used to find the region corresponding to the lumen under analysis. In the current study, besides the assessment concerning the suitability of the method to detect the lumen boundary of coronary arteries in IVUS images using the same parameters as were established for the MR images, the method has been improved in order to enhance its robustness, efficiency, automaticity and competency. Hence, a new approach was developed to identify and remove side branches of bifurcation regions so only the regions that potentially correspond to the lumen are selected for further processing, avoiding bifurcation parts at the border of the image and enhancing therefore, the robustness of the method. Moreover, the regions at the border are now discarded before computing the circularity index of each potential lumen region, which boosts the performance of the method. Additionally, noisy regions are now automatically eliminated without the use of any predefined parameter, which increases the automaticity. Finally, a post-processing step has now been added to smooth the contour found for the lumen region, which leads to better segmentation results.

The remainder of the article is organized as follow: Section 2 presents the previous studies related to the segmentation of IVUS images. Section 3 provides a detailed description of the proposed automatic lumen segmentation method. The results of the segmentation, as well as the comparison with the manual delineations and related methods found in the literature, are given in Section 4. The advantages and limitations of the proposed method are pointed out in Section 5. Finally, the conclusions are drawn up in Section 6.

\section{Previous studies}

Several studies have been proposed to address the segmentation of the lumen and media-adventitia in IVUS images acquired using different ranges of frequencies and with several artefacts that hamper the segmentation successful. In summary, the studies can be divided into approaches based on machine learning, probabilistic functions, deformable models, region growing, thresholding and morphological operations.

Lo Vercio et al. (2016) proposed a learning machine-based approach to segment the lumen and media-adventitia regions in IVUS images of coronary arteries. From the input images, a set of fea- tures obtained using noise-reduction filters, texture and edge detector operators was acquired and used as input to a Support Vector Machine (SVM) classifier, which classify the likelihood of the pixels to belong to the lumen and background regions. Hence, the classification between lumen and non-lumen, as well as between background and non-background, was performed to determine the correct location of the lumen and media-adventitia contours.

Another learning machine-based approach to segment the lumen and media-adventitia contours in IVUS images of coronary arteries was tackled by Su et al. (2016). Two Artificial Neural Networks (ANN) are used to classify the pixels inside a region of interest (ROI) as belonging to the media-adventitia region. The first ANN is used to perform the initial classification, whereas the second one performs the classification of the binary image resultant from the first ANN in order to remove noises and refine the results. Then, the pixels inside the region corresponding to the identified media-adventitia are classified as lumen and non-lumen by using the same ANNs. The contours of the regions are then submitted to the Snake active contour model proposed by Kass et al. (1988) in order to adjust them to the true boundary of each region.

A study carried out by Destrempes et al. (2014) proposed a fast-marching method (FMM) to segment the lumen and mediaadventitia boundaries in IVUS images of coronary arteries. The proposed method relies on the minimization of a function that uses the gradient of the image and the probability of the pixels to belong to a region corresponding to one of the structures of the coronary artery: lumen, media, adventitia, and surrounding tissues.

Mendizabal-Ruiz et al. (2013) proposed an approach to find the lumen contour in IVUS images of coronary arteries based on probabilistic functions. After the transformation of the IVUS images into the polar coordinates domain, the probabilities of the pixels belonging to the lumen region are used to find the correct contour by means of the minimization of a cost function. The probabilities are calculated by using a sigmoid function and an SVM classifier that receives texture features from Laws filters, which are used to deal with pixel intensities that increase due to the catheters with higher frequencies.

A parametric active contour model for the segmentation of lumen and media-adventitia contours in IVUS images of coronary arteries was proposed by Vard et al. (2012). For the lumen contour segmentation, a method based on the short-term autocorrelation (STA) is used to remove speckle noise from the lumen region. The proposed STA method, called normalized cumulative STA (NCSTA), is then used to produce a new grayscale IVUS image without noise in the lumen region. The image resulting from the NCSTA method generates a pressure force for a parametric active contour model that is used to find the lumen contour.

A 3D-FMM method was proposed by Cardinal et al. (2006) to segment the lumen and media-adventitia contours in IVUS images of femoral arteries. The 3D-FMM method is based on the Rayleigh probability density function (PDF) and a gradient function to find the correct contour of each region, i.e. lumen and media-adventitia, by means of the refinement of contours manually defined. Another study tackled by Cardinal et al. (2010) combines the PDF and gradient intensity obtained from the input images in order to calculate the propagation speed of the 3D-FMM. In addition, the proposed method automatically detects the initial contours of the lumen and media-adventitia borders to be used by the 3D-FMM method.

Taki et al. (2008) proposed a method based on thresholding and deformable models to identify the contours of the lumen and media-adventitia regions in IVUS images. Regarding the lumen contour identification, the evaluation of the pixels in the polar coordinates domain is made in order to identify the ones with intensities higher than a pre-defined threshold $T$. If $I(r, \theta)>T$, then the pixel is assumed to belong to the lumen border. The initial contour of the lumen is then submitted to a parametric active contour and 
a geometric model to adjust it to the true borders of the corresponding lumen region.

The combination of fuzzy clustering with morphological operation was proposed by dos Santos et al. (2005) to identify the lumen region in IVUS images. The fuzzy clustering defined with two clusters was applied to the images after the transformation into the polar coordinates domain. Then, the morphological closing operation was performed to obtain more regular borders. The extracted contour was converted into the Cartesian coordinate domain and overlapped with the IVUS images to present the final segmentation result. Segmentation methods based on the Otsu's threshold algorithm and morphological operations for the identification of the lumen region in IVUS image were also proposed by Sofian et al. (2015) and Moraes and Furuie (2011).

Most of the above mentioned studies still required manual interventions, re-training of the learning-based model and the transformation of the image into the polar coordinates domain. In addition, the use of threshold values as proposed by Taki et al. (2008) can fail to identify the initial contour of the lumen in different datasets due to the usual variability of the grayscale intensities. Hence, the development of a more robust, efficient, automated and less complex solution was the goal of this study.

\section{Materials and methods}

\subsection{IVUS images used}

This study was accomplished by using the IVUS images of coronary arteries selected for the IVUS Segmentation Challenge described in Balocco et al. (2014) that were kindly provided by the authors. The images were acquired using a Si5 imaging system (Volcano Corporation) equipped with an Eagle Eye catheter operating at a frequency of $20 \mathrm{MHz}$ (Balocco et al., 2014). A total of 326 images with ground truths provided were selected for validating the proposed method. The manual delineations of the contours corresponding to the lumen and media-adventitia regions were performed by two experts, and one of them repeated the manual delineations about one week after the first delineations (Balocco et al., 2014). The images have a resolution of $384 \times 384$ pixels and the pixel size is $0.026 \mathrm{~mm} \times 0.026 \mathrm{~mm}$. More details about the IVUS images are available in Balocco et al. (2014).

\subsection{Proposed method}

The proposed lumen segmentation method is made up of three main stages: pre-processing, segmentation and lumen identification. The diagram of the proposed method is shown in Fig. 1.

The pre-processing stage is necessary to minimize noise and adjust the contrast of the input image. Then, the enhanced image is submitted to the segmentation stage in order to separate the regions with low pixel values, which include the background and the lumen. Relatively to the initial version (Jodas et al., 2016), the method has three new steps in this stage: the Gaussian pyramid, which is adopted to reduce the resolution of the input image; the elimination of regions at the border of the image; and the identification and removal of side branches at the bifurcation regions. Additionally, connected component labelling is employed here to separate all regions of the binary image corresponding to the low intensity values instead of using the region growing algorithm proposed in the initial version. The lumen identification stage uses two classification indexes to identify the region corresponding to the lumen of the artery under analysis, which is then inputted to an active contour algorithm for further refinement of the boundary. A post-processing step is now included in the method in order to smooth the contour resultant from the active contour algorithm, which leads to better segmentation results.

\subsubsection{Pre-processing}

The first step of the pre-processing stage is the use of a median filter with a mask of $5 \times 5$ to minimize the noise in the original image. The median filter was chosen due to its ability to remove noise without compromising the boundaries of the regions of interest.

The contrast enhancement step improves the brightness of the dark regions of the input image. The gamma correctionbased method proposed by Huang et al. (2013) was employed to avoid the overestimation of regions with low-level intensities. The method relies on the probability density function (PDF) and the cumulative density function (CDF) of the intensity values as:

$T(l)=l_{\max } \times\left(l / l_{\max }\right)^{1-c d f_{w}(l)}$,

where $c d f_{w}(l)$ is the weighting CDF of the intensity value $l$ and $l_{\max }$ is the highest possible intensity value. The weighting PDF $\left(p d f_{w}\right)$ and weighting $\operatorname{CDF}\left(c d f_{w}\right)$ are defined as:

$c d f_{w}(l)=\sum_{l=0}^{l_{\max }} \frac{p d f_{w}(l)}{\sum p d f_{w}}$

and

$p d f_{w}(l)=p d f_{\text {max }} \times\left(\frac{p d f(l)-p d f_{\text {min }}}{p d f_{\text {max }}-p d f_{\text {min }}}\right)^{\alpha}$,

where $p d f_{\min }$ is the minimum probability of the PDF, $p d f_{\max }$ is the maximum probability of the PDF, $\sum p d f_{w}=\sum_{l=0}^{l_{\max }} p d f_{w}(l)$ and $\alpha$ is a parameter that controls the amount of contrast enhancement. The value of the $\alpha$ parameter is determined by partitioning the PDF of the grayscale intensities as proposed in our previous work (Jodas et al., 2016):

$\alpha=\sum_{i=1}^{t} P D F_{\min _{i}}-\sum_{j=t+1}^{N} P D F_{\max _{j}}$,

where $P D F_{\min }$ and $P D F_{\max }$ represent the probabilities of the low and high intensities of the input image, respectively, $t$ is the value obtained by the Otsu's threshold algorithm and $N$ is the highest possible intensity value. Here, if the value of $\alpha$ is less than 0 (zero), the contrast correction is not necessary since there are more pixels with high intensities.

\subsubsection{Segmentation}

The K-means clustering algorithm is a well-known method to separate regions with similar characteristics (of intensity, for instance) in images. Finding the correct cluster centroids to be used in the K-means algorithm is a challenging task because different images have different cluster centroids. Subtractive clustering (Bataineh et al., 2011; Chiu, 1994) has been proposed as an alternative approach to find the adequate number of cluster centroids based on the potential of each pixel in the neighbourhood. The advantage of the subtractive clustering algorithm is that the cluster centroids do not change in different runs. This is due to the fact that the potential function relies on the pixel values only (or on another feature calculated from the pixels of the input image).

Although subtractive clustering has been widely used in image segmentation and classification problems, the computational complexity of $O\left(d^{2}, N^{2}\right)$, with $N$ representing the number of data points and $d$ the dimensional number, has limited its application to large-scale problems (Sun et al., 2012). Hence, a reduction in the number of data points, i.e. pixels, is necessary to reduce the computational time of the subtractive clustering algorithm. Here, the Gaussian pyramid is applied to the input IVUS image in order to reduce its resolution and consequently, the number of pixels to be used in the subtractive clustering algorithm.

The Gaussian pyramid is an image processing technique used to reduce the resolution of images in repeated steps 


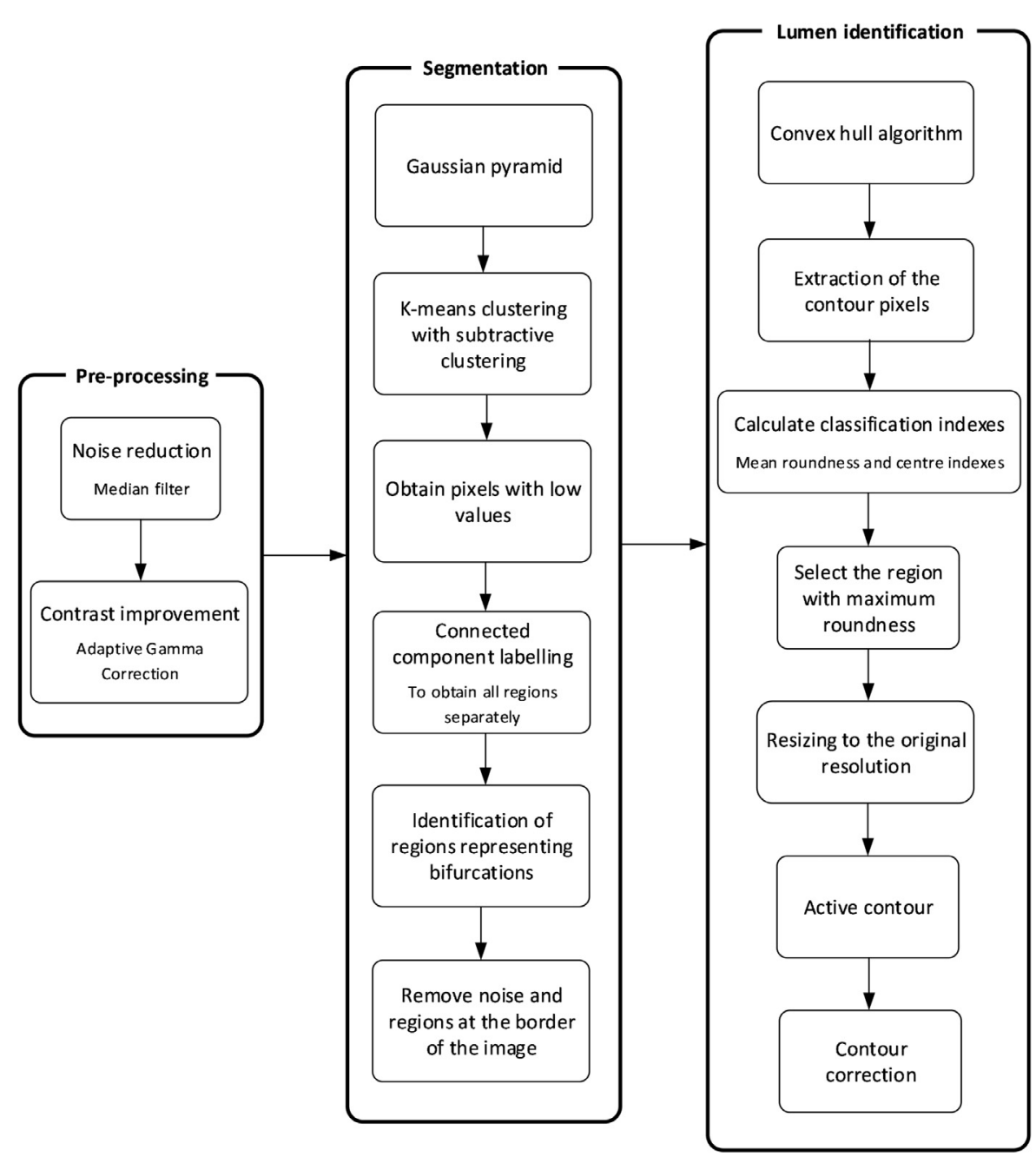

Fig. 1. Diagram of the proposed method.

(Adelson et al., 1984). Pattern recognition, texture analysis and image compression are examples for the use of the Gaussian pyramid. The pyramid is a sequence of copies of the original image generated by recursively smoothing the image with a low-pass filter and reducing its resolution by half. The following equation represents the basis of the Gaussian pyramid:

$g_{l}(i, j)=\sum_{m=-2}^{2} \sum_{n=-2}^{2} w(m, n) g_{l-1}(2 i+m, 2 j+n)$,

where $l$ is a level of the pyramid, $i$ and $j$ are the pixel coordinates at the $l$ th level, $g_{l-1}$ is the smoothed image at the level $l-1$ and $w(m, n)$ is a $5 \times 5$ low-pass filter applied to the neighbourhood of the pixel $(i, j)$. The original image is represented by $g_{0}$, i.e. the image at the level $l=0$. The image at the level $l=1$ is generated by smoothing $g_{0}$ with $w(m, n)$ followed by downsampling the resulting image by a factor of two. The image at the level $l=2$ is obtained by applying the same procedure on the image $g_{1}$, i.e. the image at the level $l=1$. The process is repeated until the desired number of levels is reached. The final result is a pyramid in which the base is the original image and the top represents the smoothed image with the smallest resolution. The smoothing of the image is necessary to avoid the aliasing effect generated when the resolution is reduced. An example of the application of the Gaussian pyramid in order to reduce the resolution of an IVUS image and accelerate the use of the subtractive clustering algorithm is shown in Fig. 2.
Although the most common use of the Gaussian pyramid is compressing an input image and multiscale processing at lower resolutions, here the goal is to generate a pyramid of lower resolution images for the input image in order to select the one with the smallest resolution, i.e. the image at the top of the pyramid, where the subtractive clustering algorithm is then applied with lower computational time than if it was applied on the original image. Here, the number of reductions performed by the Gaussian pyramid is defined as equal to 2, leading to a pyramid with three levels in which the base is represented by the input IVUS image with $384 \times 384$ pixels of resolution and the top represents the IVUS image with the smallest resolution of $96 \times 96$ pixels that is inputted to the subtractive clustering algorithm. Thereafter, the subsequent steps of the proposed method are performed on the image with the smallest resolution in order to obtain a binary image with the identified lumen, which is then restored to the input image resolution without performing any processing on the higher resolution images of the pyramid. The choice of the Gaussian pyramid was due to its simple implementation and ability to reduce the input image resolution without losing important information about the structure of interest.

After the downsampling of the input image, the K-means algorithm with subtractive clustering suggested in Dhanachandra et al. (2015) is employed to separate the regions of the image according to the grayscale intensity. The centroid of each cluster is found by means of the subtractive clustering algorithm. After the centroids 

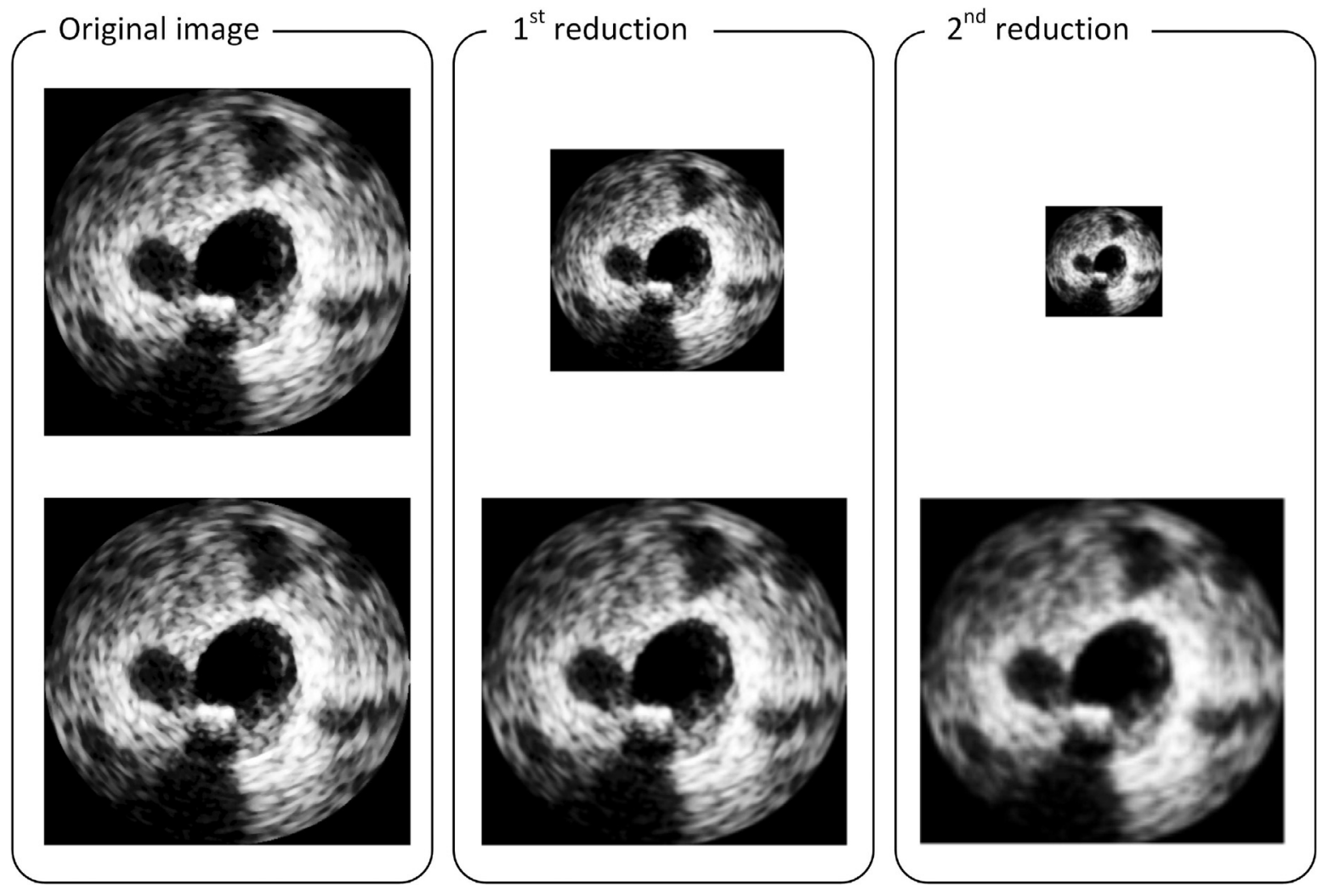

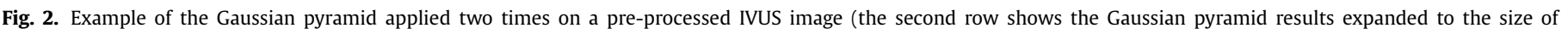
the original image).

have been found, the K-means clustering is applied to separate the regions of the image. As proposed in our previous study, four clusters are used here to correctly identify the region corresponding to the lumen. Since the lumen and background regions have low intensities in IVUS images, the same approach employed is used to find the cluster having the regions with low-intensity pixels for identifying the one representing the correct lumen. A binary image with such regions is returned, in which the identified regions are represented by white pixels and the background by black pixels. The image is then submitted to the connected component labelling algorithm in order to obtain all regions of the image separately. Here, the connected component labelling is performed on the image corresponding to the cluster with low-intensity values to obtain a set with all regions of interest. Firstly, the algorithm performs a pixel-by-pixel scan of the binary image from the top to bottom and left to right in order to identify a white pixel and assign a label to it that relies on the evaluation of the adjacent pixels that share the same intensity. If none of the neighbour pixels have until then been labelled, a new label is assigned to the pixel under analysis. Otherwise, the pixel under analysis receives a label assigned to a neighbour pixel. The process continues until all pixels of the binary image have been labelled. Then, pixels having the same label are merged to form a single region. Thereafter, each region is used as the input to the lumen identification stage in order to find the one that represents the lumen.

\subsubsection{Lumen identification}

In our previous work, a set of measures was used to evaluate each region of interest obtained by the region growing algorithm in order to identify the one corresponding to the lumen in the MR images of carotid arteries. Hence, the mean roundness (MR), irregularity (Ir) and centre $(d)$ indexes are included in the following circularity function, which is used to evaluate each region of the binary image:

$$
E=M R+\frac{1}{I r}+\frac{1}{d}
$$

The MR was proposed to determine the circularity of objects in images (Ritter and Cooper, 2009). It consists of calculating the ratio between the average radius and the distance between the radius of each border pixel of the object and the average radius. The mean roundness is calculated according to:

$M R=\frac{1}{N} \sum_{i=1}^{N} \frac{\overline{r_{b}}}{\left|r_{i}-\overline{r_{b}}\right|+\overline{r_{b}}}$,

where $N$ is the number of pixels of the contour of the object under analysis, $\overline{r_{b}}$ is the average radius of the object, and $r_{i}$ is the radius at the contour pixel $i$. The larger the mean roundness $(M R)$ index is, the more circular the object under analysis is.

The following irregularity index is used to avoid regions with irregular contours:

Ir $=P *\left(\frac{1}{S D}-\frac{1}{G D}\right)$,

where $P$ is the number of pixels of the contour, $S D$ is the shortest diameter and GD is the greatest diameter (Jain et al., 2015).

A centre index is also used to identify the correct location of the lumen. As described in our previous work, the lumen is a circular-shaped region located close to the centre of the input MR image. Hence, the distances between the centre of the image and the centre of each region are calculated and used to penalize those far from the centre of the MR image. The same concept can also be applied here due to the fact that the lumen is also located close to the centre of an IVUS image.

The segmentation of the lumen close to bifurcations represents a challenge due to the extension of the low-intensity values from 


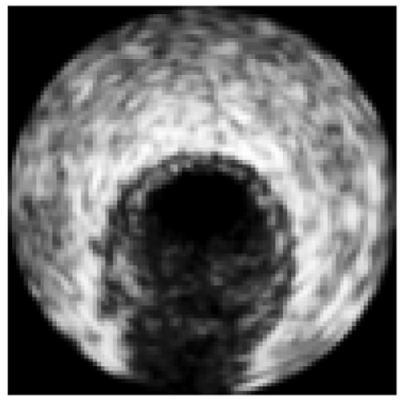

(a)

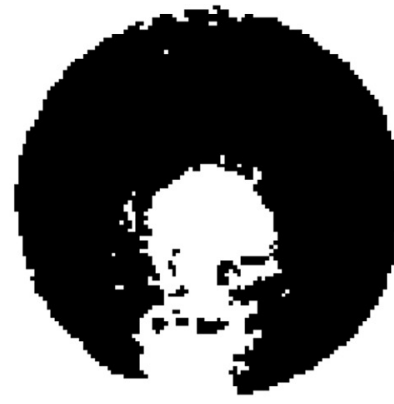

(b)

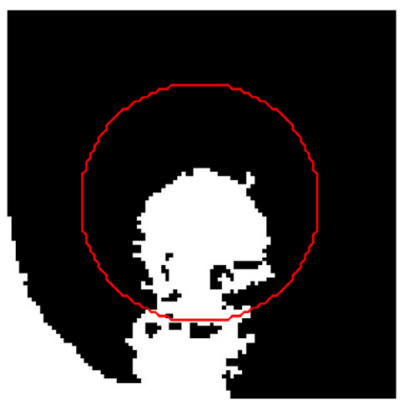

(c)

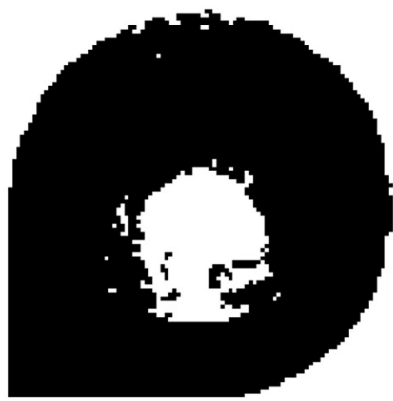

(d)

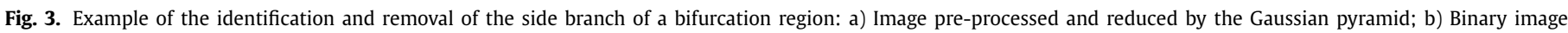

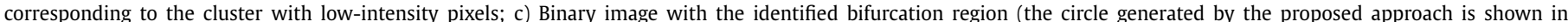

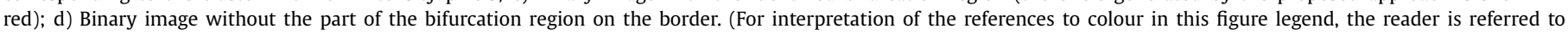
the web version of this article.)

the lumen region to the border of the input image, which causes the establishment of a region representing this extension when the cluster corresponding to the low-intensity values is built. Hence, in the initial version of the proposed method, these regions were not assessed in the subsequent processing steps, since the regions at the border of the image are discarded before the identification of the lumen. Therefore, a new approach was developed to identify regions representing bifurcations in order to distinguish these regions from the ones at the border of the binary images corresponding to low-intensity values. The proposed approach is based on the generation of circles surrounding the regions of possible lumens, like the approach proposed in de Macedo et al. (2016). However, here, the distances from the centres of the input image to the pixels of the bifurcation regions at the borders are used to define the radius of the circles, instead of the distance transform suggested in de Macedo et al. (2016). Hence, the regions obtained by the connected component labelling algorithm are evaluated to identify the one that is in the centre of the input image. Then, the number of pixels of this region that are at the border are computed in order to verify if the region represents a bifurcation. Once the region is identified as a bifurcation, the distances between the centre of the image and each pixel of the region at the border is calculated. The radius of the circle is then calculated according to:

$r=\min (\operatorname{dist}(C p, B p))-\min (\operatorname{dist}(C p, R p))$,

where $C p$ is the pixel at the centre of the IVUS image, $B p$ is the set of pixels of the bifurcation region that are at the border of the image and $R p$ is the set of all pixels of the contour of the bifurcation. The circle generated is centred at the centre of the IVUS image and the region outside this circle is removed from the binary image corresponding to the low-intensity values. An example of the proposed approach applied to an IVUS image with a bifurcation region is illustrated in Fig. 3.

As depicted in Fig. 3c, the bifurcation region can be divided into two parts, i.e. one inside the circle and the other outside. Since the part inside the circle contains the regions with the possible lumen, the regions outside the circle are removed from the binary image corresponding to the low-intensity values, leading to a new binary image that is then submitted to the subsequent steps of the lumen identification stage.

Concavities, irregularities and holes inside the regions of the binary image corresponding to the cluster with low-intensity values may be present due to high intensity values inside the lumen under analysis. Since the high intensity values are identified as belonging to another cluster when the K-means algorithm is applied, such artefacts are generated in the regions of the binary image. In order to attenuate the effect of these artefacts, the convex hull algorithm is used here. The use of the convex hull algorithm instead of morphological operations is to avoid underestimation and cutting of parts of the region of interest when concavities are presented.

The convex hull of a set of points $S$ is the smallest convex polygon containing all the points of $S$ (Berg et al., 2008). The definition of convex hull relies on the concept of a convex set, which is a region defined in a way that for every pair of points $[a, b]$ belonging to a region, the line joining such points must be totally inside the region. Here, for each region present in the binary image obtained by the connected component labelling algorithm a correspondent convex hull is generated i.e., the set of pixels representing the convex polygon that includes all the white pixels of the region, before calculating the classification indexes used to find the lumen region. An example of the application of the convex hull algorithm is shown in Fig. 4.

As depicted in Fig. 4, the regions become more regular after the application of the convex hull algorithm. Hence, the irregularity index is now redundant and the function defined by Eq. (6) is therefore, simplified to:

$E=M R+\frac{1}{d}$

In the initial version of the proposed method, the regions with less than $1.5 \%$ of the total number of pixels of the input image were discarded from the lumen identification procedure, since these regions usually represent small regions associated to noise. However, this empirically defined discarding criterion is not always robust. Hence, a new approach based on the morphological opening operation was developed in order to identify and disregard the small regions associated to noise. The morphological opening operation is the erosion followed by the dilation of an image $I$ by a structuring element $S E$. When applied to binary images, the opening operation can smooth the borders of the regions represented by white pixels, split regions connected by thin bridges and remove small regions that represent noisy artefacts. The choice of the shape and size of the structuring element is usually based on the shape of the regions of interest, and it plays an important role in effectively achieving the desired results. Since the lumen is a circular-shaped region in IVUS images, a disk-shaped structuring element is used to perform the opening operation. In addition, the size of the structuring element is adaptively defined according to the approach proposed by Gao et al. (2015) and formulated as:

$S_{0}=\frac{1}{2} \sqrt{\frac{A_{r}}{\pi}}$, 


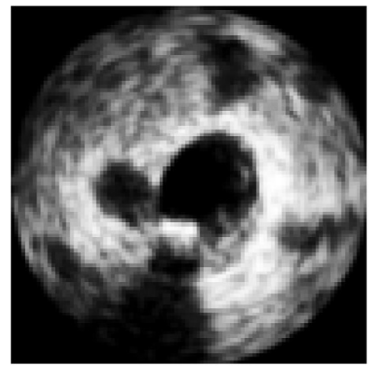

(a)

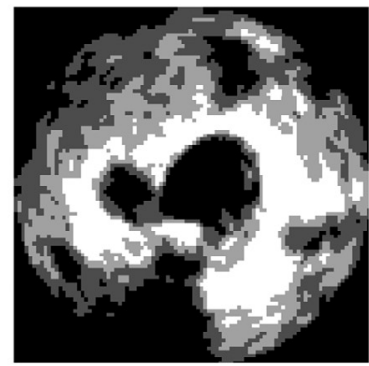

(b)

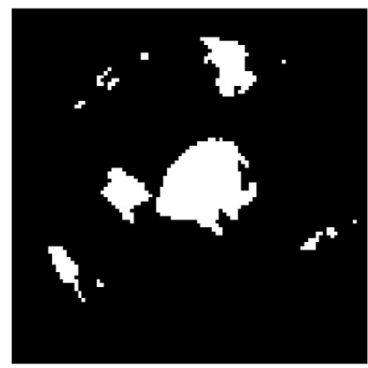

(c)

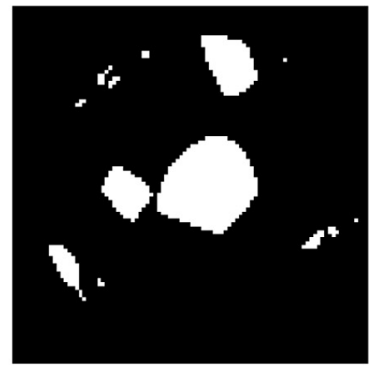

(d)

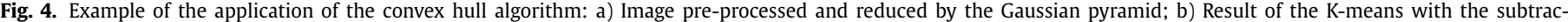

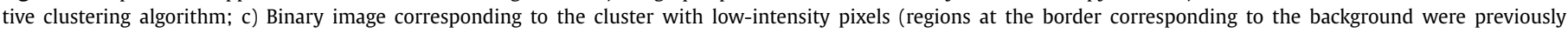
removed); d) Result of the convex hull algorithm applied to each region (white pixels) of the binary image.

where $A_{r}$ is the area of the region under analysis. The opening operation with the adaptive structuring element is applied to each region obtained by the connected component labelling algorithm. Hence, a region is not considered in the lumen identification step if its size is equal or less than the size of the structuring element, and the region is removed by the opening operation. Here, the morphological opening operation is only employed to identify small regions corresponding to noise. Therefore, the smoothing of the regions is not performed by the opening operation since the convex hull is previously applied to correct the shape of the regions under analysis. A region identified as noise and removed from the binary image by the opening operation is not selected for calculating the circularity index defined in Eq. (10).

Although the terms in Eq. (10) are defined in different contexts, the objective of using the centre index in the proposed circularity index is to penalize regions with larger values of roundness that are distant from the centre of the input image. The value of the circularity index proposed in Eq. (10) is calculated for each region resultant after the opening operation and the one with the maximum value is considered to be the lumen of the artery under study. This region is resized to the original resolution of the input IVUS image and then submitted to the Chan-Vese active contour algorithm (Chan and Vese, 2001) for further refinement of the contour. The binary image representing the lumen region may not fit the true boundary of the lumen in the IVUS image. Hence, the contour of such a region is used as the input of the Chan-Vese active contour model, which is applied to the original IVUS image in order to fit the contour to the true boundary. This refinement step plays an important role in avoiding under- or over-estimating the contour, leading the contour closer to the true boundary of the lumen under analysis and, consequently, to better results. Since the gradient of the image is not used in the Chan-Vese model, the method is recommended for the segmentation of medical images which commonly have weak boundaries of the structures of interest (Ma et al., 2010; Wang et al., 2010; Santos et al., 2013; Huang and Zeng, 2015).

The post-processing step consists in smoothing the lumen contour by using the morphological opening and dilation operations. The morphological opening operation was employed to smooth the boundaries of the lumen region generated from the result of the Chan-Vese active contour. A binary image is generated from the lumen contour such that the region inside the contour is represented by white pixels. Then, the region is smoothed by first applying the opening operation. Finally, the morphological dilation operation is performed on the resulting region in order to restore its size as close as possible to the size of the original region. The sizes of the structuring elements are defined according to the size of the region under analysis and formulated as:

$S_{o}=\frac{1}{2} \sqrt{\frac{A_{l}}{\pi}}, S_{d}=\frac{1}{N} \sum_{i=1}^{N} \sqrt{\left(C o_{i}-C r_{k}\right)^{2}}$,

where $S_{0}$ and $S_{d}$ are the sizes of the structuring element used in the morphological opening and dilation operations, respectively, $A_{l}$ is the area of the lumen region, $C_{o}$ is the contour of the region resulting from the opening operation, $C_{r}$ is contour of the region obtained from the lumen contour and $N$ is the number of pixels of the contour $C_{o} . \mathrm{Co}_{i}$ is the ith pixel of $\mathrm{Co}$, and $\mathrm{Cr}_{k}$ is the $k$ th pixel of $\mathrm{Cr}$ closest to $\mathrm{Co}_{i}$. The size $S_{0}$ was proposed by Gao et al. (2015), whereas $S_{d}$ is defined as the average distance between the contours $C_{o}$ and $C_{r}$.

The steps performed in the automatic segmentation of the lumen in one IVUS image are depicted in Fig. 5.

\subsection{Quantitative analysis}

The contours obtained by the proposed method and the related manual delineations were compared based on four measures: Jaccard measure $(J M)$, Dice coefficient $(D C)$, Hausdorff distance $(H D)$ and percentage of area difference $(P A D)$.

The Jaccard measure is calculated by means of the ratio between the size of the intersection and the size of the union of the regions corresponding to the automatic $\left(S_{\text {auto }}\right)$ and manual $\left(S_{\text {manual }}\right)$ segmentations:

$J M=\frac{\left|S_{\text {auto }} \cap S_{\text {manual }}\right|}{\left|S_{\text {auto }} \cup S_{\text {manual }}\right|}$.

The Jaccard measure is important to assess the overlap of the region identified by the proposed method with respect to the corresponding manual delineation. Similarly, the Dice coefficient is used to calculate the overlap between the automatic and manual segmentations:

$D C=\frac{2 *\left|S_{\text {auto }} \cap S_{\text {manual }}\right|}{\left|S_{\text {auto }}\right|+\left|S_{\text {manual }}\right|}$.

The Hausdorff distance is important to assess the closeness of two contours, and it is defined here as the maximum between the greatest distances between the pixels of the automatic $\left(C_{a}\right)$ and manual $\left(C_{m}\right)$ contours:

$H D\left(C_{a}, C_{m}\right)=\max \left\{\max _{a \in C a} \min _{b \in C m} d(a, b), \max _{b \in C m} \min _{a \in C a} d(a, b)\right\}$,

where $a$ and $b$ are the pixels of contours $C_{a}$ and $C_{m}$, respectively, and $d(a, b)$ is the Euclidean distance between these pixels.

The percentage of area difference $(P A D)$ represents the difference between the areas of the contour obtained from the automatic segmentation $\left(A_{\text {auto }}\right)$ and the corresponding manual delineation $\left(A_{\text {manual }}\right)$ with respect to the area of the manual delineation: 


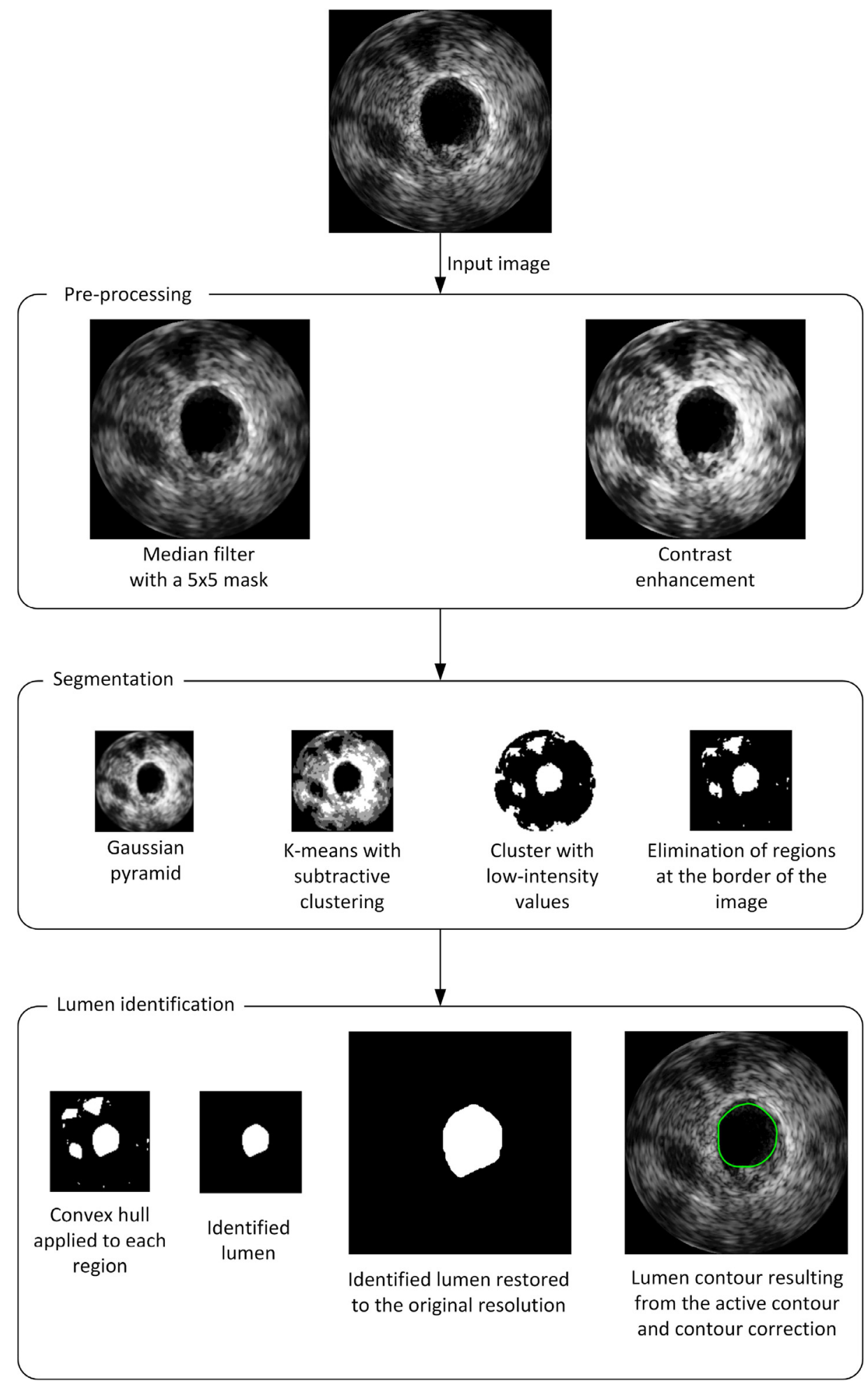

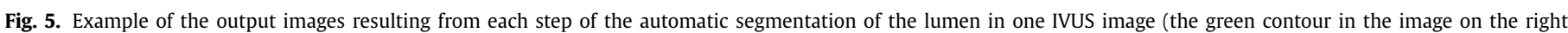

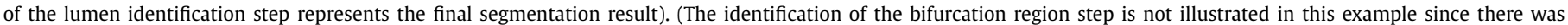
no side branch in the IVUS image used.). (For interpretation of the references to colour in this figure legend, the reader is referred to the web version of this article.)

$P A D=\frac{\left|A_{\text {auto }}-A_{\text {manual }}\right|}{A_{\text {manual }}}$.

The lumen area and the average lumen diameter of the automatic and manual segmentations were also calculated to compare the segmentation results by means of the regression analysis and
Bland-Altman analysis. The lumen area is the area inside the contour of the lumen and the average lumen diameter $(A L D)$ is defined as:

$A L D=\frac{1}{N} \sum_{i=1}^{N} 2 * r_{i}$, 
Table 1

Details about the datasets and the validation measures of the studies used to compare the segmentation results of the proposed method.

\begin{tabular}{llll}
\hline Authors & Catheter's frequency & Number of frames & Validation measure(s) \\
\hline Lo Vercio et al. (2016) & $20 \mathrm{MHz}$ & 149 & $J M$ and PAD \\
Su et al. (2016) & $20 \mathrm{MHz}$ & 461 & $J M$ and $H D$ \\
Sofian et al. (2015) & $20 \mathrm{MHz}$ & 30 & $J M, H D, P A D$ and $D C$ \\
Destrempes et al. (2014) & $40 \mathrm{MHz}$ & 3207 & $H D$ \\
Mendizabal-Ruiz et al. (2013) & $20 \mathrm{MHz}$ and $40 \mathrm{MHz}$ & 585 & $J M, H D$ and $D C$ \\
Cardinal et al. (2010) & $20 \mathrm{MHz}$ & 1593 & $H D$ \\
Vard et al. (2012) & $30 \mathrm{MHz}$ & 40 & $H D$ \\
Taki et al. (2008) & $30 \mathrm{MHz}$ & 60 & $H D$ \\
\hline
\end{tabular}

*JM = Jaccard measure; $H D=$ Hausdorff distance; $P A D=$ percentage of area difference; $D C=$ Dice coefficient; $\mathrm{MHz}=$ Megahertz

where $N$ is the number of pixels of the contour of the lumen and $r_{i}$ is the radius of the $i$ th pixel.

\section{Results}

The proposed method was implemented in MATLAB software (The Mathworks Inc., Natick, USA) and executed in a desktop computer equipped with an Intel i7-4700 HQ processor $(2.4 \mathrm{GHz})$ and 16GB of RAM memory. A comparison between the contours obtained from the proposed method and those generated by manual delineations was performed in order to evaluate the accuracy of the segmentation results. In addition, the results of the proposed method were also compared with the ones reported in related studies found in the literature. The details about the datasets and the validation measures of the studies used here to compare the segmentation results of the new automatic segmentation method are shown in Table 1.

Since there are not any common validation measures in the studies indicated in Table 1, all measures described in Section 3.3 were calculated and the ones used in each of these studies were selected to compare the performance of the proposed automatic lumen segmentation.

The proposed automatic lumen segmentation was also compared with the results obtained from the eight participant groups of the IVUS Segmentation Challenge held in the 2011 Computing and Visualization for (Intra)Vascular Imaging (CVII) workshop of the Medical Image Computing \& Computer Assisted Intervention (MICCAI) conference.

The results of the proposed method with the contrast enhancement activated and deactivated were also taken into account in order to evaluate the improvement obtained by adjusting the contrast of the input image.

\subsection{Initialization of the parameters}

The following parameters were defined to perform the automatic segmentation: the size of the mask of the median filter was set to $5 \times 5$, which proved to be the most suitable template for removing noise without affecting the edges of the lumen; when the contrast enhancement was necessary, the value of the $\alpha$ parameter was defined as the difference between the probability of the low and high intensities of the image, as described by Eq. (4); the number of reductions performed by the Gaussian pyramid was automatically defined as equal to 2, leading to images with a resolution of $96 \times 96$ to be processed by the K-means with subtractive clustering; the radius $r_{a}$ and $r_{b}$ of the subtractive clustering algorithm were set to 1.2 and 1.8 , respectively; and the number of iterations of the Chan-Vese active contour algorithm was set to 500, which was higher when compared to our previous study using MR images (200 iterations) due to the higher resolution of the IVUS images under analysis.

\subsection{Performance of the proposed method}

Examples of the segmentation results and corresponding manual delineations are shown in Fig. 6.

In Fig. 6, the green contours are the results obtained by the proposed method, whereas the blue, red and yellow contours represent the related manual delineations. The lumen was correctly identified in all images and is very close to the corresponding manual delineations. Due to the subjective analysis of each expert, the automatically segmented contour is very close to corresponding manual delineation in some cases, while small differences were produced in other cases as shown in Fig. $6 \mathrm{~b}$.

The effect of the post-processing step on the lumen contour resulting from the Chan-Vese active contour is shown in Fig. 7.

As shown in Fig. 7a, the automatically segmented lumen contour can be somewhat irregular and leak from the true boundary as depicted in the image of the third row. However, the lumen contours became more regular and smooth after the final postprocessing step, which leads to results very similar to the manual delineations (Fig. 7c).

The effectiveness of the proposed approach to identify and eliminate side branches of bifurcation regions is apparent in Fig. 8.

As shown in Fig. 8b, the segmentation errors resulting from the automatic lumen segmentation are due to the removal of the whole bifurcation region from the binary image representing the low-intensity values before applying the circularity index in Eq. (10), leading to the identification of another region as belonging to the potential lumen. However, as shown in Fig. 8c, the lumen was successfully segmented after applying the proposed approach to identify and split the bifurcation region.

From the 326 IVUS images used in the experiments, the proposed method successfully segmented 324 images. The average values of the Jaccard measure, Hausdorff distance, percentage of area difference and Dice coefficient of the automatically segmented lumen in comparison with the three manual delineations of the correctly segmented images are indicated in Tables 2 and 3. Additionally, the average values of the same four measures obtained from the intra- and inter-observer analysis are indicated in Table 4.

As shown in Tables 2 and 3, the average values of the Jaccard measure, percentage of area difference and Dice coefficient for the manual delineations 2 and 3 are better than those obtained in comparison to the manual delineation 1 . However, the results of the Hausdorff distance are similar to almost all the manual delineations, except in the case where the adjustment of the contrast of the images was not applied (Table 2), leading to a decrease of the Hausdorff distance for the manual delineation 2. As shown in Table 3, the average value of the Jaccard measure increased from $0.87 \pm 0.07$ to $0.88 \pm 0.06$ after the application of the contrast enhancement. Additionally, an increase of the average value of the Dice coefficient from $0.93 \pm 0.04$ to $0.94 \pm 0.04$ was also obtained after adjusting the contrast of the images. The Hausdorff distance 



(a)
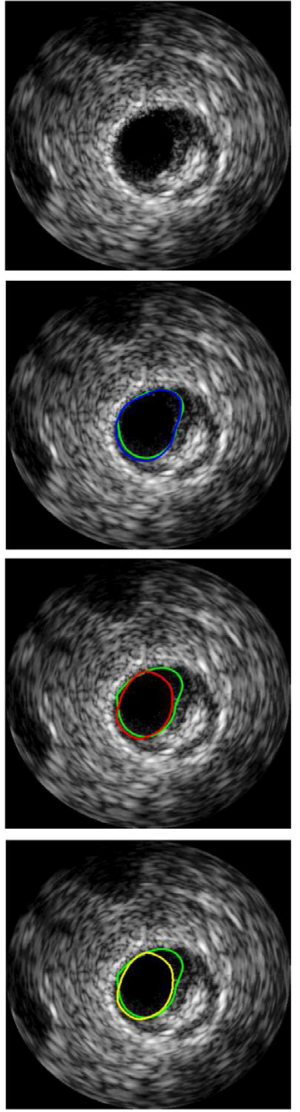

(b)
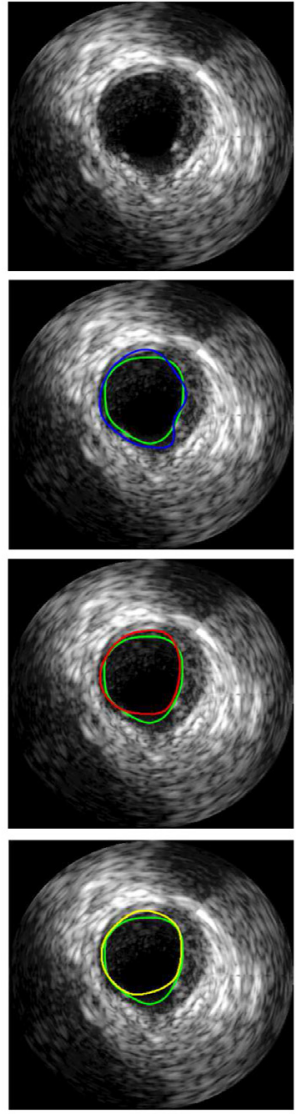

(c)
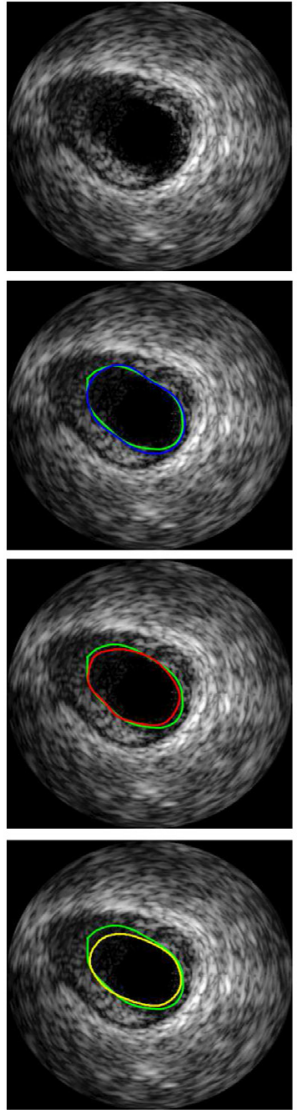

(d)
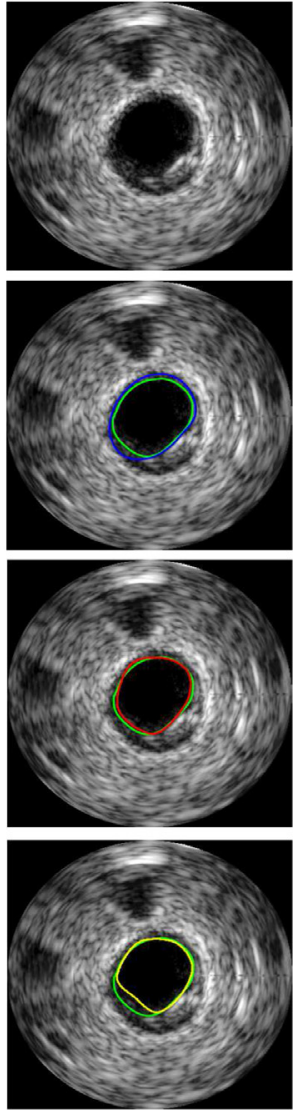

(e)

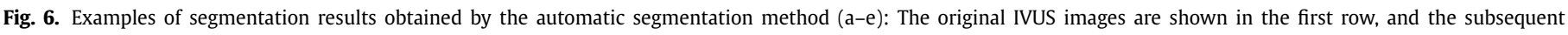

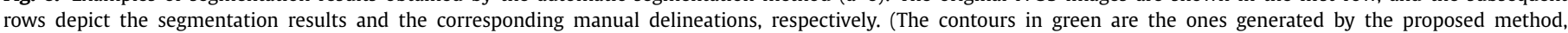

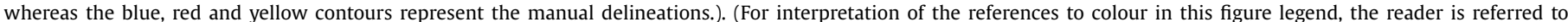
the web version of this article.)

Table 2

Average values of the Jaccard measure (JM), Hausdorff distance (HD), Percentage of area difference $(P A D)$ and Dice coefficient $(D C)$ obtained by the proposed method without the application of the contrast enhancement.

\begin{tabular}{lllll}
\hline & Manual delineation 1 & Manual delineation 2 & Manual delineation 3 & Average \\
\hline JM & $0.86 \pm 0.07$ & $0.88 \pm 0.07$ & $0.88 \pm 0.07$ & $\mathbf{0 . 8 7} \pm \mathbf{0 . 0 7}$ \\
$H D$ & $0.30 \pm 0.17$ & $0.29 \pm 0.18$ & $0.30 \pm 0.19$ & $\mathbf{0 . 3 0} \pm \mathbf{0 . 1 8}$ \\
$P A D$ & $0.12 \pm 0.08$ & $0.08 \pm 0.07$ & $0.08 \pm 0.08$ & $\mathbf{0 . 0 9} \pm \mathbf{0 . 0 8}$ \\
$D C$ & $0.92 \pm 0.05$ & $0.93 \pm 0.04$ & $0.93 \pm 0.04$ & $\mathbf{0 . 9 3} \pm \mathbf{0 . 0 4}$ \\
\hline
\end{tabular}

*The values of the Hausdorff distance $(H D)$ are presented in millimeters; Manual delineation 1 represents the tracing done by the expert 1 , whereas the manual delineations 2 and 3 are the first and second tracings of the expert 2 , respectively.

Table 3

Average values of the Jaccard measure (JM), Hausdorff distance (HD), Percentage of area difference $(P A D)$ and Dice coefficient $(D C)$ obtained by the proposed method with the application of the contrast enhancement.

\begin{tabular}{lllll}
\hline & Manual delineation 1 & Manual delineation 2 & Manual delineation 3 & Average \\
\hline$J M$ & $0.87 \pm 0.06$ & $0.88 \pm 0.06$ & $0.88 \pm 0.06$ & $\mathbf{0 . 8 8} \pm \mathbf{0 . 0 6}$ \\
$H D$ & $0.29 \pm 0.15$ & $0.29 \pm 0.17$ & $0.29 \pm 0.18$ & $\mathbf{0 . 2 9} \pm \mathbf{0 . 1 7}$ \\
$P A D$ & $0.11 \pm 0.07$ & $0.08 \pm 0.07$ & $0.08 \pm 0.08$ & $\mathbf{0 . 0 9} \pm \mathbf{0 . 0 7}$ \\
$D C$ & $0.93 \pm 0.04$ & $0.94 \pm 0.04$ & $0.94 \pm 0.04$ & $\mathbf{0 . 9 4} \pm \mathbf{0 . 0 4}$ \\
\hline
\end{tabular}

*The values of the Hausdorff distance $(H D)$ are presented in millimeters; Manual delineation 1 represents the tracing done by the expert 1, whereas the manual delineations 2 and 3 are the first and second tracings of the expert 2 , respectively. 

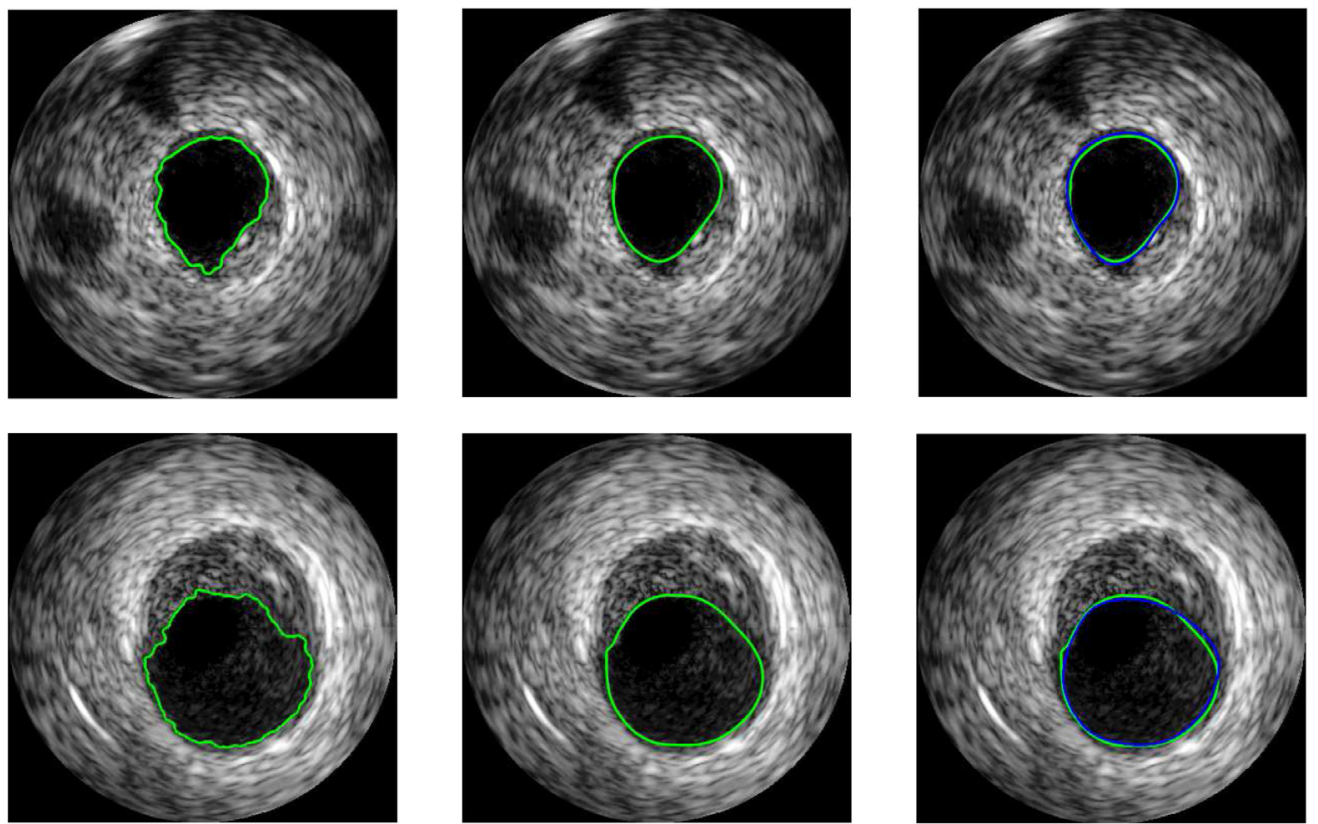

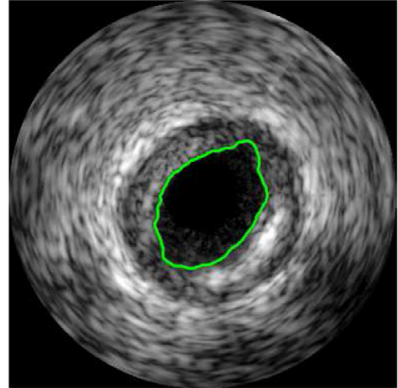

(a)

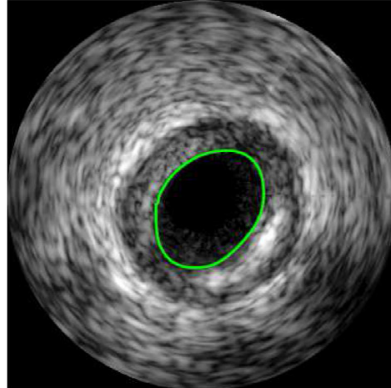

(b)

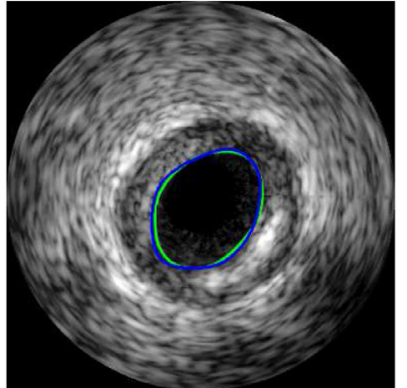

(c)

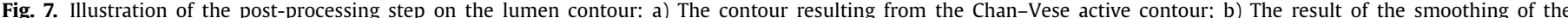

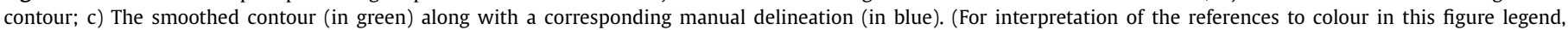
the reader is referred to the web version of this article.)

Table 4

Average values of the Jaccard measure (JM), Hausdorff distance $(H D)$, Percentage of area difference $(P A D)$ and Dice coefficient $(D C)$ obtained from the intra- and inter-observer analysis.

\begin{tabular}{lllll}
\hline & Exp 1 vs Exp 2 (1st) & Exp 1 vs Exp 2 (2nd) & Exp 2 (1st) vs Exp 2 (2nd) & Average \\
\hline JM & $0.88 \pm 0.05$ & $0.87 \pm 0.05$ & $0.93 \pm 0.05$ & $\mathbf{0 . 8 9} \pm \mathbf{0 . 0 5}$ \\
HD & $0.28 \pm 0.13$ & $0.30 \pm 0.13$ & $0.17 \pm 0.13$ & $\mathbf{0 . 2 5} \pm \mathbf{0 . 1 3}$ \\
PAD & $0.11 \pm 0.08$ & $0.13 \pm 0.08$ & $0.04 \pm 0.05$ & $\mathbf{0 . 0 9} \pm \mathbf{0 . 0 7}$ \\
DC & $0.94 \pm 0.03$ & $0.93 \pm 0.03$ & $0.96 \pm 0.03$ & $\mathbf{0 . 9 4} \pm \mathbf{0 . 0 3}$
\end{tabular}

*Exp stands for Expert; 1st and 2nd indicate the first and second delineations of Expert 2, respectively; the values of the Hausdorff distance $(H D)$ are expressed in millimeters.

also decreased from $0.30 \pm 0.18 \mathrm{~mm}$ to $0.29 \pm 0.17 \mathrm{~mm}$ after adjusting the contrast of the images. In terms of the intra- and interobserver variability, the results of Table 4 show that the proposed method is also in accordance with the average values computed from the comparison between Expert 1 and Expert 2. The average values of the Jaccard measure, Hausdorff distance, percentage of area difference and Dice coefficient between both experts were $0.89 \pm 0.05,0.25 \pm 0.13 \mathrm{~mm}, 0.09 \pm 0.07$ and $0.94 \pm 0.03$, respectively, which are very similar to the results obtained by our method (see Tables 2 and 3).

The mean values of the lumen area and average lumen diameter calculated for each manual delineation and for the corresponding automatically detected lumen are shown in Table 5 .
The mean lumen area obtained from the proposed method was $7.93 \pm 3.63 \mathrm{~mm}^{2}$, which is close to the one obtained from the manual delineation $3\left(7.94 \pm 3.55 \mathrm{~mm}^{2}\right)$. Additionally, the mean value of the average lumen diameter of the automatically detected lumen was also close to the one obtained from the manual delineation 3 (3.07 $\pm 0.71 \mathrm{~mm}$ and $3.08 \pm 0.68 \mathrm{~mm}$, respectively). Although the results of the proposed method were closer to the manual delineation 3, no significant differences were found between the areas and average diameters of the automatically detected and manually delineated lumen contours.

A comparison between the proposed automatic lumen segmentation and the methods reported in the literature was also carried out, which led to the results shown in Tables 6 and 7. 

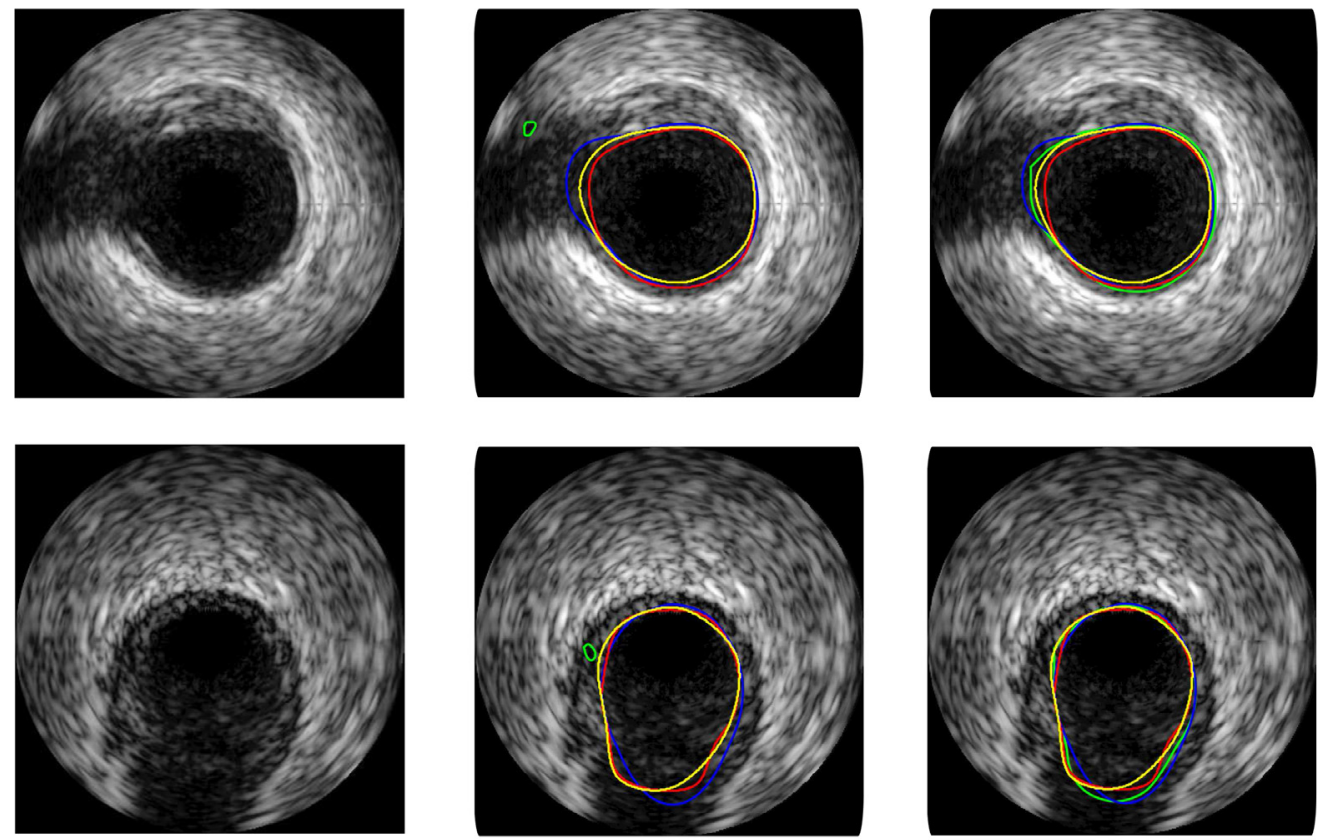

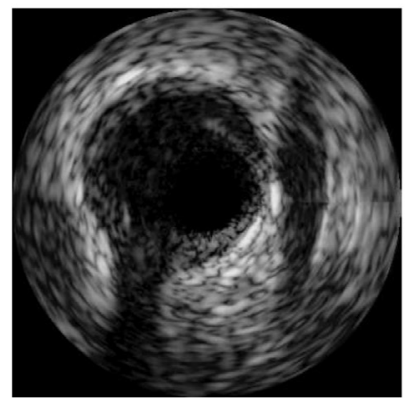

(a)

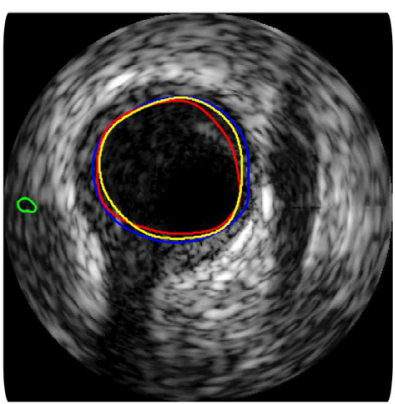

(b)

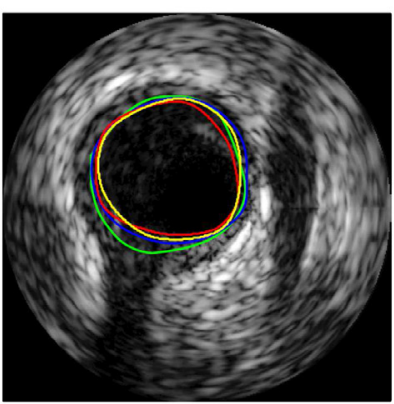

(c)

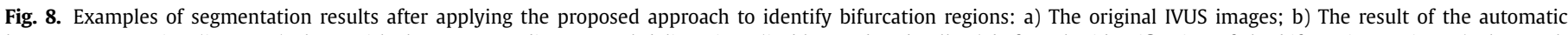

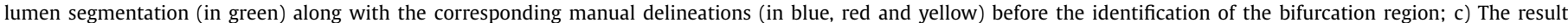

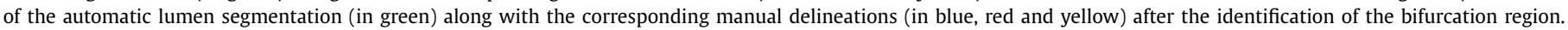
(For interpretation of the references to colour in this figure legend, the reader is referred to the web version of this article.)

Table 5

Mean values of the lumen area $(L A)$ and average lumen diameter $(A L D)$ for the manual delineations and the automatically segmented lumen after adjusting the contrast of the images.

\begin{tabular}{lllll}
\hline & Manual delin. 1 & Manual delin. 2 & Manual delin. 3 & Proposed method \\
\hline Mean $L A\left(\mathbf{m m}^{2}\right)$ & $8.75 \pm 3.73$ & $8.03 \pm 3.56$ & $7.94 \pm 3.55$ & $7.93 \pm 3.63$ \\
Mean ALD $(\mathbf{m m})$ & $3.11 \pm 0.63$ & $3.10 \pm 0.68$ & $3.08 \pm 0.68$ & $3.07 \pm 0.71$
\end{tabular}

*Manual delin. 1 represents the delineation done by Expert 1, whereas manual delin. 2 and manual delin. 3 are the first and second delineations of Expert 2, respectively.

Table 6 shows the average measures of the general performance calculated from each participant group of the IVUS Segmentation Challenge proposed at the MICCAI 2011 CVII workshop (Balocco et al., 2014). Table 6 demonstrates that the method proposed by Participant group 3 outperformed all the others presented at the challenge. The performance of our method is comparable to the one proposed by this group. Regarding the results obtained after adjusting the contrast of the input images, the average value of the Jaccard measure obtained from the proposed automatic lumen segmentation was $0.88 \pm 0.06$ against $0.88 \pm 0.05$ presented by Participant group 3 at the IVUS Segmentation Challenge; the average Hausdorff distance of our method was $0.29 \pm 0.17 \mathrm{~mm}$ against $0.34 \pm 0.14 \mathrm{~mm}$ of this participant group; and the average percentage of area difference obtained from our method was $0.09 \pm$ 0.07 against $0.06 \pm 0.05$ of the same group.
Table 7 shows the comparison between our automatic lumen segmentation approach and related methods proposed in the literature. The comparison shows that our method is in accordance with the ones proposed in the studies found, achieving results comparable or even better than the ones of those methods.

The computational cost of the most important procedures in the segmentation and identification stages of the proposed method when applied to each image of the dataset used in this study is shown in Table 8.

The execution time of the Chan-Vese active contour is the highest among the other algorithms involved, representing about 68\% of the total time. The K-means with subtractive clustering represents about $27 \%$ of the total time, whereas the smoothing of the contour takes only $6 \%$ of the whole segmentation and identification of the lumen. 
Table 6

Jaccard measure $(J M)$, Hausdorff distance $(H D)$ and percentage of area difference $(P A D)$ obtained from the general performance of the IVUS Segmentation Challenge for the test images acquired at $20 \mathrm{MHz}$ (Balocco et al., 2014).

\begin{tabular}{llllllll}
\hline & P1 & P2 & P3 & P4 & P5 & P7 & P8 \\
\hline$J M$ & $0.81 \pm 0.12$ & $0.83 \pm 0.08$ & $\mathbf{0 . 8 8} \pm \mathbf{0 . 0 5}$ & $0.77 \pm 0.09$ & $0.79 \pm 0.08$ & $0.84 \pm 0.08$ & $0.81 \pm 0.09$ \\
$H D$ & $0.47 \pm 0.39$ & $0.51 \pm 0.25$ & $\mathbf{0 . 3 4} \pm \mathbf{0 . 1 4}$ & $0.47 \pm 0.22$ & $0.46 \pm 0.30$ & $0.38 \pm 0.26$ & $0.42 \pm 0.22$ \\
$P A D$ & $0.14 \pm 0.13$ & $0.14 \pm 0.12$ & $\mathbf{0 . 0 6} \pm \mathbf{0 . 0 5}$ & $0.15 \pm 0.12$ & $0.16 \pm 0.09$ & $0.11 \pm 0.12$ & $0.11 \pm 0.11$
\end{tabular}

*P stands for the participant group; Participant group 6 (P6) did not perform the segmentation of the lumen contour and it explains the absence of the average values of this participant group; the values of the Hausdorff $(H D)$ distance are presented in millimeters.

Table 7

Average measures obtained from the proposed method and the related ones found in the literature.

\begin{tabular}{|c|c|c|c|c|}
\hline Authors & $J M$ & $H D$ & $P A D$ & $D C$ \\
\hline Lo Vercio et al. (2016) & $0.8300 \pm 0.0500$ & - & $0.1800 \pm 0.0600$ & - \\
\hline Su et al. (2016) & 0.9182 & 0.2243 & - & - \\
\hline Sofian et al. (2015) & $0.8624 \pm 0.0193$ & $0.5444 \pm 0.1290$ & $0.0645 \pm 0.0509$ & $0.9260 \pm 0.0111$ \\
\hline Destrempes et al. (2014) & - & $0.3300 \pm 0.0700$ & - & - \\
\hline Mendizabal-Ruiz et al. (2013) & $0.8671 \pm 0.0341$ & $0.1398 \pm 0.0384$ & - & $0.9283 \pm 0.0201$ \\
\hline Vard et al. (2012) & - & $0.3044 \pm 0.1853$ & - & - \\
\hline Cardinal et al. $(2010)^{\mathrm{b}}$ & - & $0.4300 \pm 0.3000$ & - & - \\
\hline Taki et al. (2008) & - & $0.7081 \pm 0.2491$ & - & - \\
\hline Proposed method & $0.8800 \pm 0.0600$ & $0.2900 \pm 0.1700$ & $0.0900 \pm 0.0700$ & $0.9400 \pm 0.0400$ \\
\hline
\end{tabular}

Table 8

Computational cost of the most important procedures of the proposed method when applied to each image (in seconds).

\begin{tabular}{lllll}
\hline & K-means & Chan-Vese active contour & Smoothing of the contour & Total time \\
\hline Average & 1.5279 & 3.8694 & 0.3192 & 5.7165 \\
Std & 0.2322 & 1.1645 & 0.1452 & 1.5419 \\
\hline
\end{tabular}

*Std $=$ Standard deviation

Linear regression analysis for the lumen area and average lumen diameter showed a high correlation between the automatic segmentations and manual delineations, as shown in Figs. 9 and 10.

For the lumen area, the correlation coefficients of the proposed method compared to the manual delineations 1,2 and 3 were $0.985096,0.975378$ and 0.974440 , respectively, before adjusting the contrast of the input images; while for the case when the contrast adjustment was applied, the correlation coefficients were $0.985729,0.974483$ and 0.974314 , respectively. A slight improvement in the correlation coefficients between the automatic segmentations and the manual delineations of Expert 1 was obtained after the application of the contrast enhancement. Regarding the average lumen diameter, the correlation coefficients between the automatic segmentations and manual delineations 1, 2 and 3 were $0.977080,0.972486$ and 0.971514 , respectively, before adjusting the contrast of the input images. When the contrast enhancement was applied, the correlation coefficients were 0.977346, 0.972354 and 0.971756 , respectively. Similar to the lumen area, a slight improvement in the correlation coefficients of the average lumen diameter between the automatic segmentations and manual delineations 1 and 3 was also achieved after adjusting the contrast of the input images.

The difference between the area calculated by the automatically segmented lumen and the ones obtained from the three manual delineations is depicted in Fig. 11 by Bland-Altman plots. For the average lumen diameter, the difference between the automatic and manual segmentations is represented in Fig. 12.

Fig. 11 shows a significant underestimation of the lumen area of the automatic segmentation compared to the manual delin- eation 1, leading to average differences of $-0.89136 \mathrm{~mm}^{2}$ and $-0.82174 \mathrm{~mm}^{2}$ before and after adjusting the contrast of the input images, respectively. In contrast, the average difference between the automatically segmented lumen and the manual delineations 2 and 3 is small. The average differences of the lumen area between the automatically segmented lumen and the manual delineation 2 were $-0.16389 \mathrm{~mm}^{2}$ and $-0.09497 \mathrm{~mm}^{2}$ before and after adjusting the contrast of the images, respectively. For the manual delineation 3 , the average differences were $-0.07421 \mathrm{~mm}^{2}$ and $-0.00808 \mathrm{~mm}^{2}$ before and after the adjustment of the contrast, respectively.

Regarding the average lumen diameter, a slight average difference was found between the automatic and manual segmentations. For the manual delineation 1 , the average differences were $-0.05160 \mathrm{~mm}$ and $-0.03283 \mathrm{~mm}$ before and after the contrast enhancement of the input images, respectively; for the manual delineation 2 , the average differences were $-0.04473 \mathrm{~mm}$ and $-0.02680 \mathrm{~mm}$ before and after the contrast enhancement, respectively; and for the manual delineation 3 , the average differences were $-0.02701 \mathrm{~mm}$ and $-0.00963 \mathrm{~mm}$ before and after the contrast enhancement, respectively.

The linear regression and Bland-Altman analysis obtained from the delineations of the two experts is depicted in Fig. 13.

\section{Discussion}

The development of automatic segmentation methods applied to medical images plays an important role in providing experts with auxiliary diagnosis tools for identifying various types of pathological conditions. For example, the segmentation of the lumen and media-adventitia regions in IVUS images represents an 
Without contrast enhancement

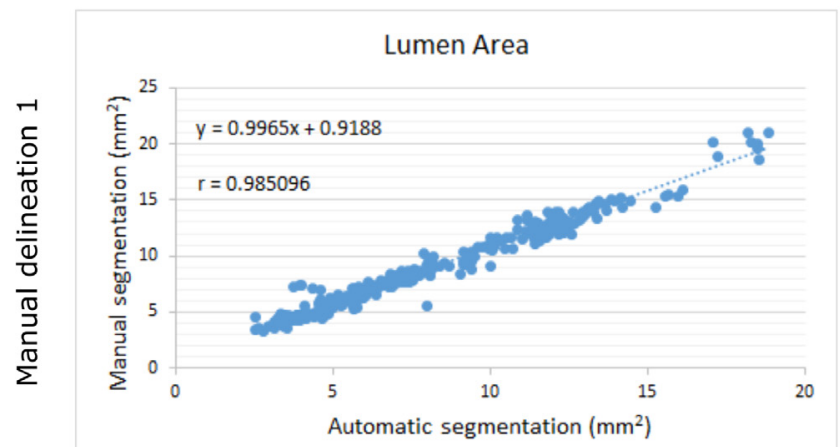

Lumen Area
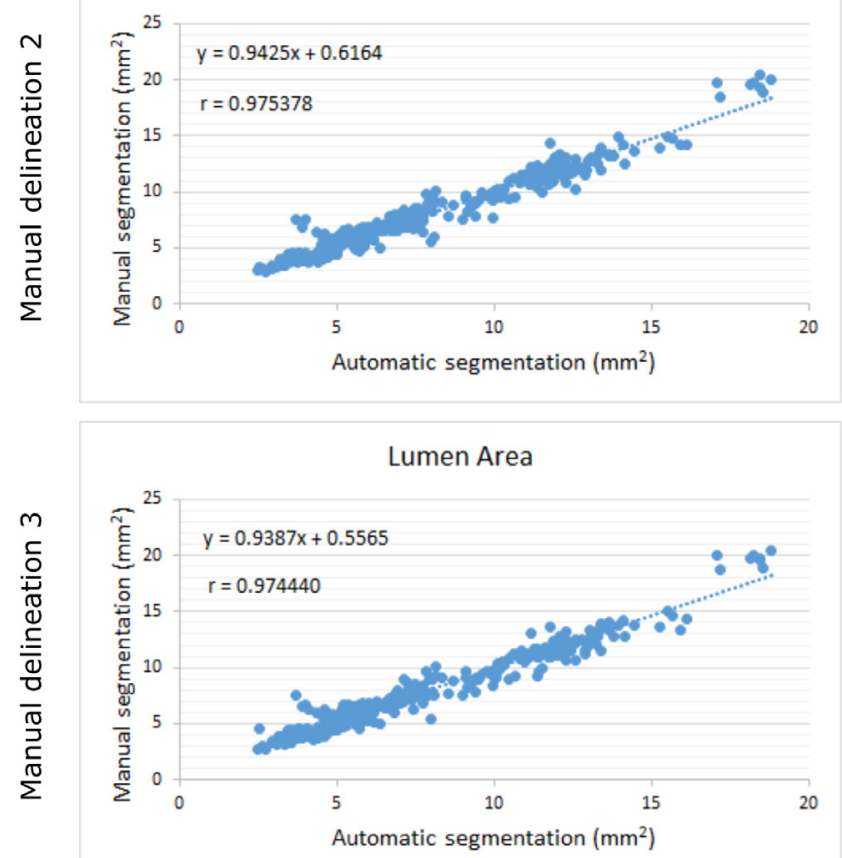

With contrast enhancement
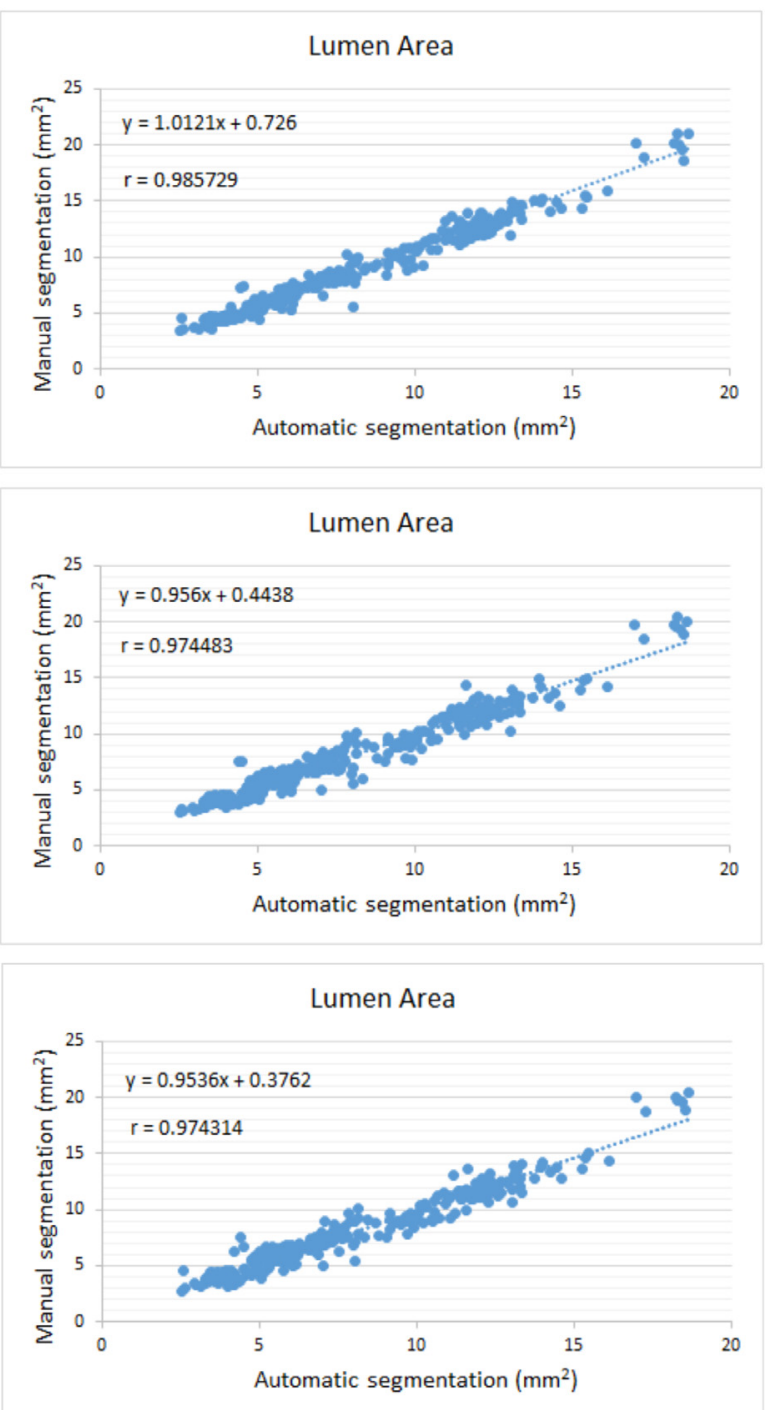

Fig. 9. Area of the automatically segmented lumen versus the three corresponding manual delineations before and after adjusting the contrast of the input images.

important step to quickly identify and quantify possible atherosclerosis in arteries.

The automatic segmentation of the lumen region in IVUS images of coronary arteries was successfully tackled in this study. The use of unsupervised classification and circularity index to identify the lumen region is the kernel of the proposed method. In our previous work, the initial version of the proposed method was applied to identify the lumen region in MR images of carotid arteries. Based on the fact that the lumen is a circular-shaped region with low-intensity values in axial black-blood MR images, the use of a circularity index was proposed to identify the region corresponding to the correct lumen among those obtained from the subtractive clustering algorithm. One goal of this study was to assess the viability of the method for segmenting the lumen in IVUS images of coronary arteries. Additionally, improvements were developed to make the method more robust, flexible, efficient and competent. Briefly, the original empirical criterion concerning noise was redefined, side branches in bifurcations regions are now successfully tackle, and the segmentation accuracy has been considerably improved.

\subsection{Initialization of the parameters}

The values of the parameters adopted in our previous study concerning MR images were also employed here to show the ability of the proposed method in segmenting the lumen in IVUS images. The mask of the median-filter used has also been used in many studies related to medical image segmentation; which therefore, indicates that it is a suitable choice to attenuate the noise present in the input images without leading to excessive smoothing of the borders of the structures of interest. The value of the $\alpha$ parameter in Eq. (2) is automatically calculated by using the difference between the probabilities of the low and high grayscale intensities of the input image as given by Eq. (4). Hence, the adjustment of the contrast is not necessary if more pixels with high grayscale intensities are present in the input image. The number of clusters that are defined has an important role in the proposed method. In most cases, the grayscale intensity of the lumen in IVUS images is well-defined and distinguishable from other structures in the images. Hence, the number of clusters initially proposed in our previous study is also suitable in the segmentation of the lumen in IVUS images. On using the subtractive clustering algorithm, the $r_{a}$ and $r_{b}$ parameters may affect the number of clusters to be gener- 
Without contrast enhancement
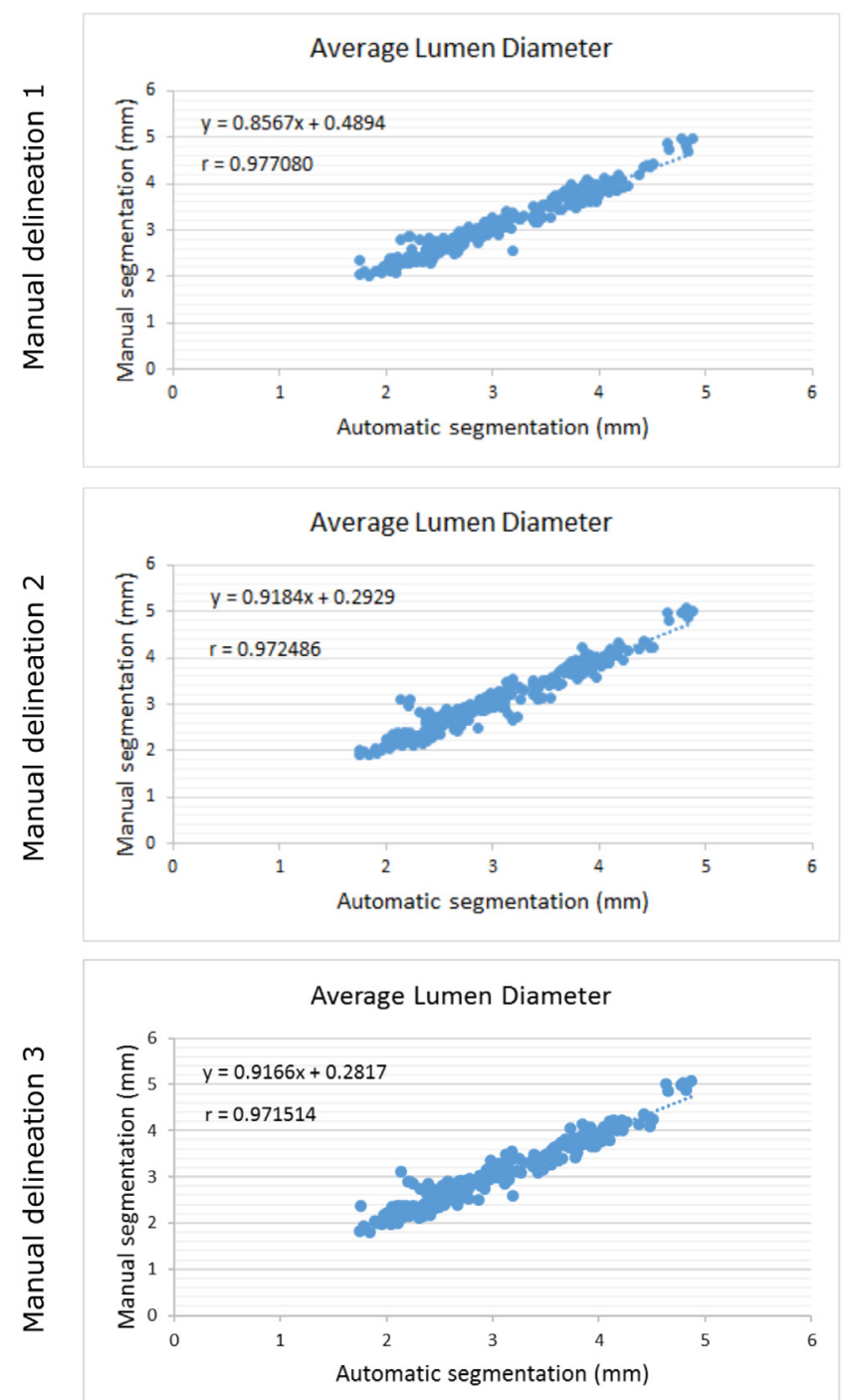

With contrast enhancement

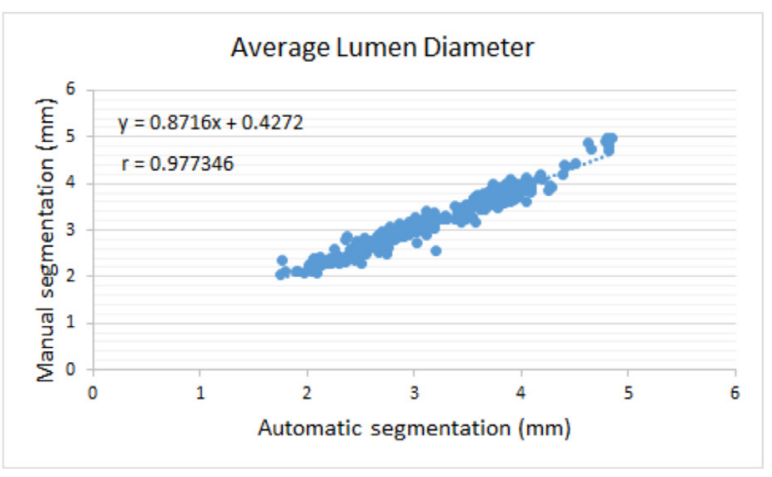

Average Lumen Diameter

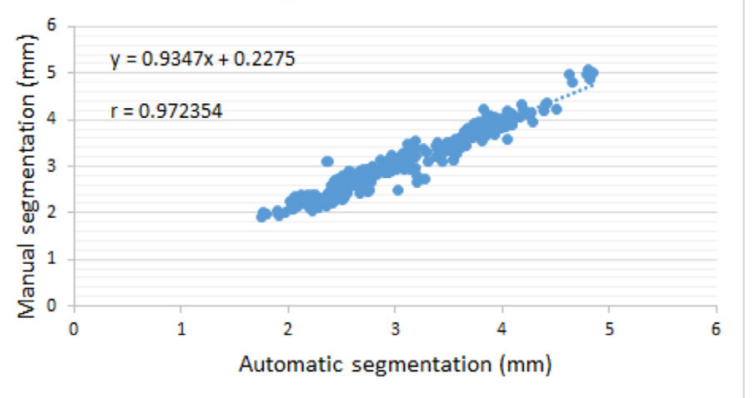

Average Lumen Diameter

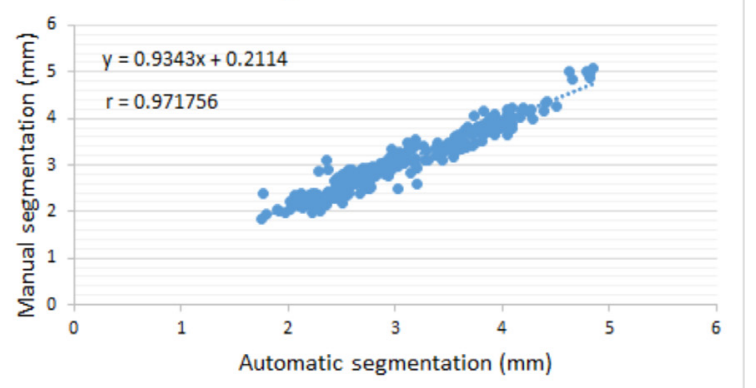

Fig. 10. Average lumen diameter of the automatically segmented lumen versus the three manual delineations before and after adjusting the contrast of the input images.

ated (Chiu, 1994). However, since the subtractive clustering is only used to generate the centroids of the expected number of clusters, the values of these parameters can also be used to separate the regions in IVUS images according to the same expected number. Regarding the downsampling of the resolution of the input IVUS image, the Gaussian pyramid was implemented in such way that it is automatically performed until the total number of pixels of the input image is equal or less than 22,500 , which was found as appropriate to reduce the computational cost of the subtractive clustering algorithm. Since all the IVUS images used have the same resolution $(384 \times 384)$, the downsampling process was performed twice, leading to a final image resolution of $96 \times 96$ (9216 pixels).

\subsection{Performance of the proposed method}

When compared to the related studies found in the literature, the proposed automatic segmentation of the lumen does not require any kind of user interaction and is easily implemented without using complex algorithms. The K-means with subtractive clustering is used to separate the regions of the input IVUS image according to the expected number of clusters. The subtractive clustering is only used as a prior step to find the appropriate initial centroids to be used in the K-means clustering algorithm. Different results could be produced when the centroids are randomly selected and used in the K-means clustering algorithm. Since the result of the K-means clustering algorithm depends on the selection of the initial centroids, we decided to use the subtractive clustering due to its stability to find the same initial centroids even when the algorithm is executed several times. Additionally, the subtractive clustering algorithm is easily implemented, although the computational time increases in images with higher resolution. Once the initial centroids are found, the traditional K-means clustering algorithm is applied to the input image.

Because the low-intensity values are associated to the lumen and background regions, the connected component labelling algorithm is applied to obtain all regions of the cluster belonging to the low-intensity values as a binary image. The regions corresponding to the background of the IVUS image are at the border of the generated binary image. In our previous work, a term representing the number of pixels of the region at the border of the image was added to reduce its circularity index. Here, the region is simply discarded from the binary image to avoid additional processing. Additionally, the morphological opening operation with adaptive size of the structuring element is proposed to remove noisy 
Without contrast enhancement
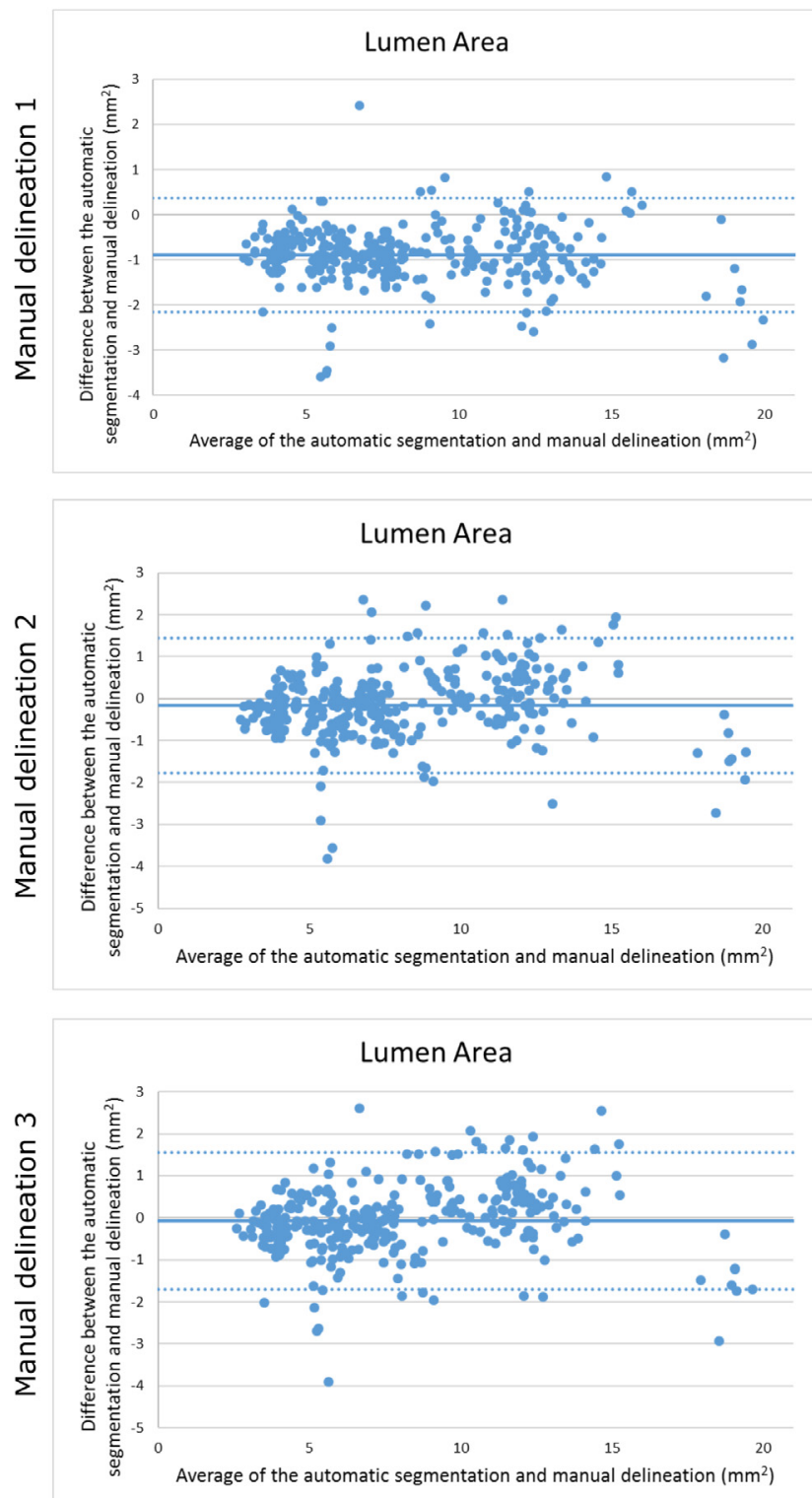

With contrast enhancement
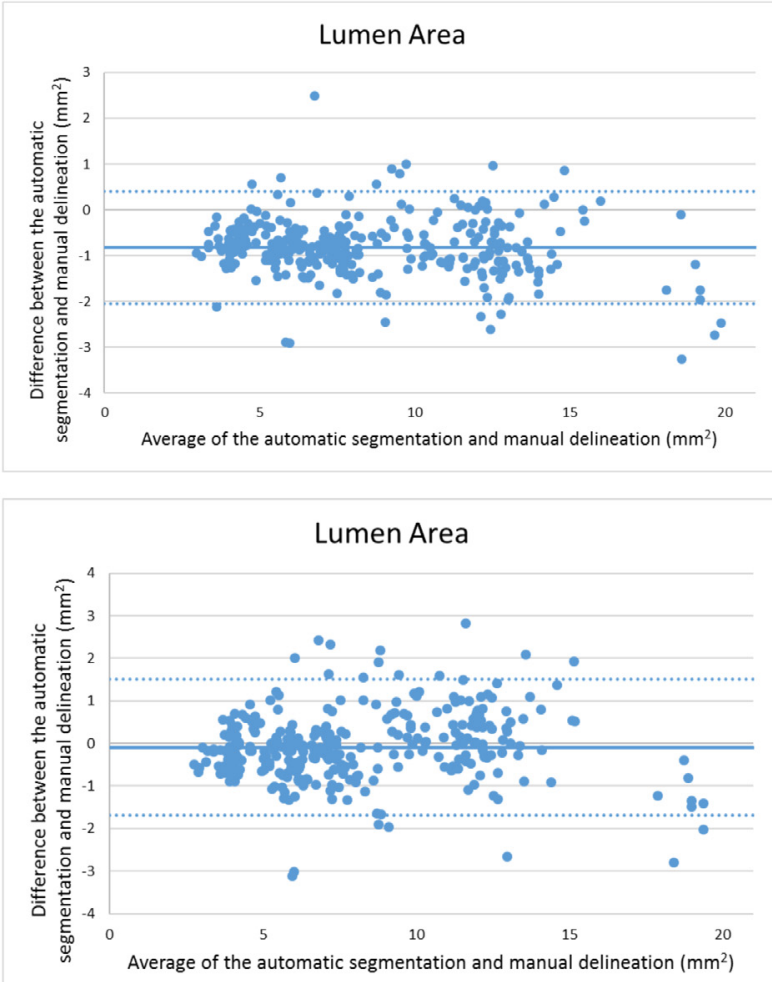

Lumen Area

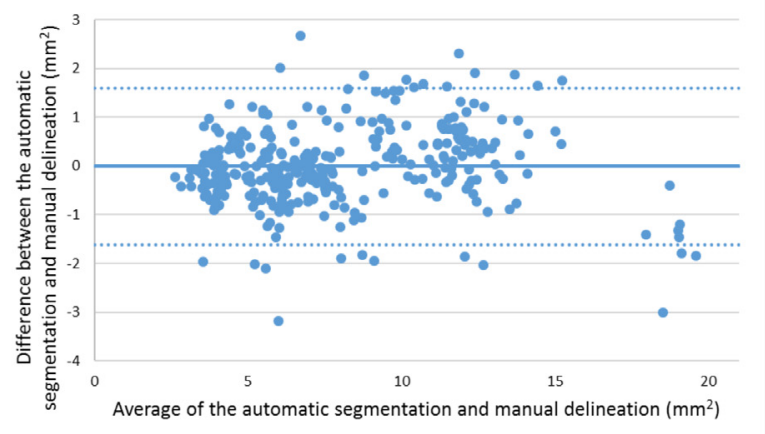

Fig. 11. Bland-Altman plots of the lumen area of the automatically segmented lumen and the three corresponding manual delineations.

artefacts without the need of a heuristic criterion as in the initial version. The morphological operations in the new post-processing step of the proposed method revealed that they were effective in smoothing the lumen contour resulting from the Chan-Vese active contour and removing possible irregularities.

An important contribution of this study is an effective approach to identify regions corresponding to side branches in the input IVUS images. The low-intensity values of the side branch extend from the lumen region to the border of the IVUS images when a bifurcation is presented. Hence, the bifurcation is represented by a single region with pixels at the border of the input image when the cluster with low-intensity values is generated by the K-means clustering algorithm. In order to avoid the elimination of this region in the lumen identification step, an approach based on the delimitation of the potential lumen region by a circle centred on the centre of the input IVUS image is proposed to separate and remove the branch of the bifurcation region. The proposed approach proved to be effective in eliminating the side branches of bifurca- tion regions and therefore, in decreasing the number of erroneous segmentations.

The average values of the measures described in Section 3.3 were obtained from the 324 IVUS images successfully segmented by the proposed method. The contrast enhancement of the IVUS images can improve the brightness of the region corresponding to the intima layer, allowing a better distinction between this region and the lumen. Hence, the segmentation accuracy obtained before and after adjusting the contrast of the input image were compared. A slight improvement in the average values of the Jaccard measure and Dice coefficient was found when the contrast enhancement was applied. The average value of the Jaccard measure was $0.87 \pm 0.07$ before adjusting the contrast of the images, whereas a slight increasing to $0.88 \pm 0.06$ was achieved after the application of the contrast. Additionally, the Hausdorff distance decreased from $0.30 \pm 0.18 \mathrm{~mm}$ to $0.29 \pm$ $0.17 \mathrm{~mm}$ after adjusting the contrast. Regarding the Bland-Altman analysis, the average differences between the area calculated from the proposed method and the ones obtained from the manual 
Without contrast enhancement
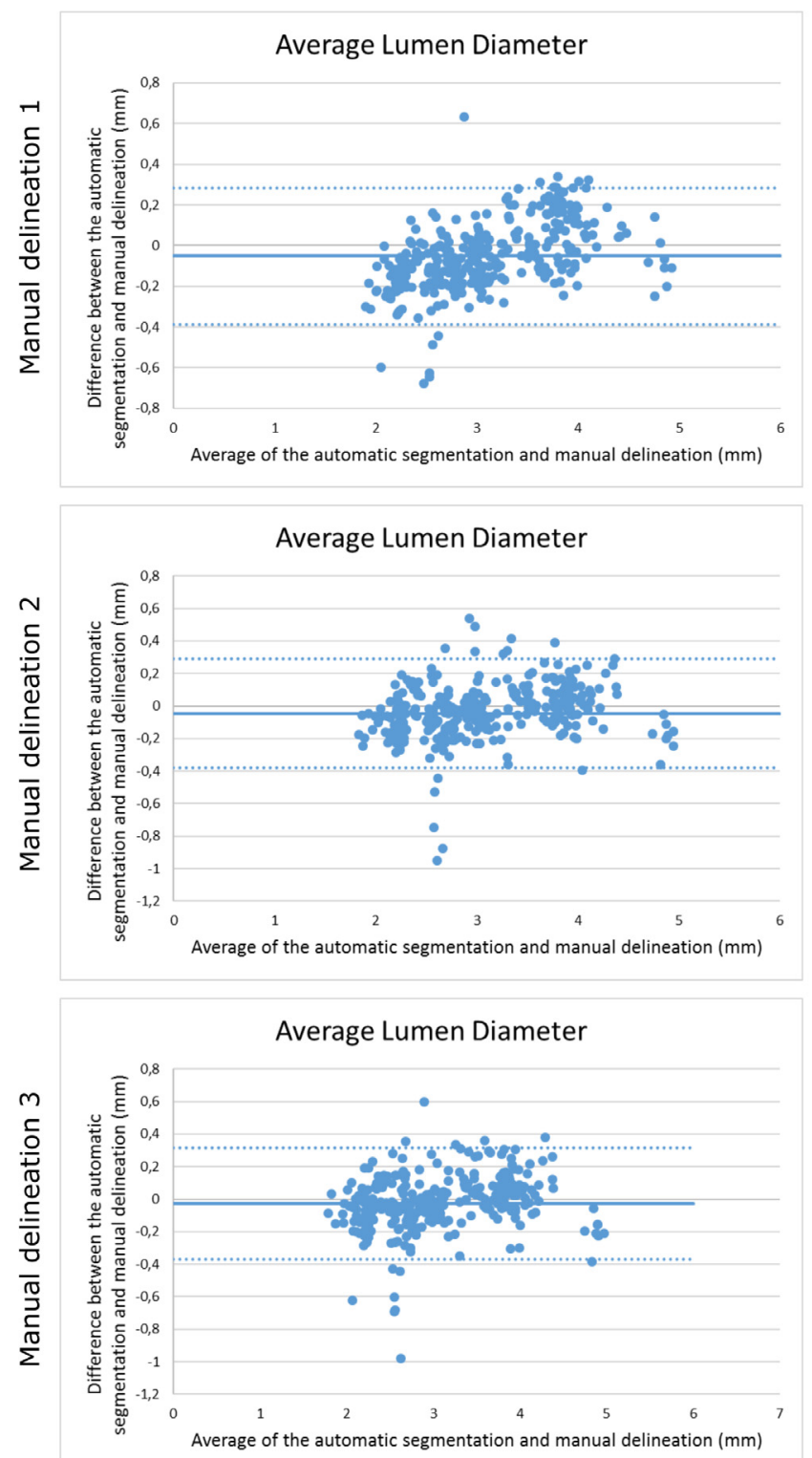

With contrast enhancement
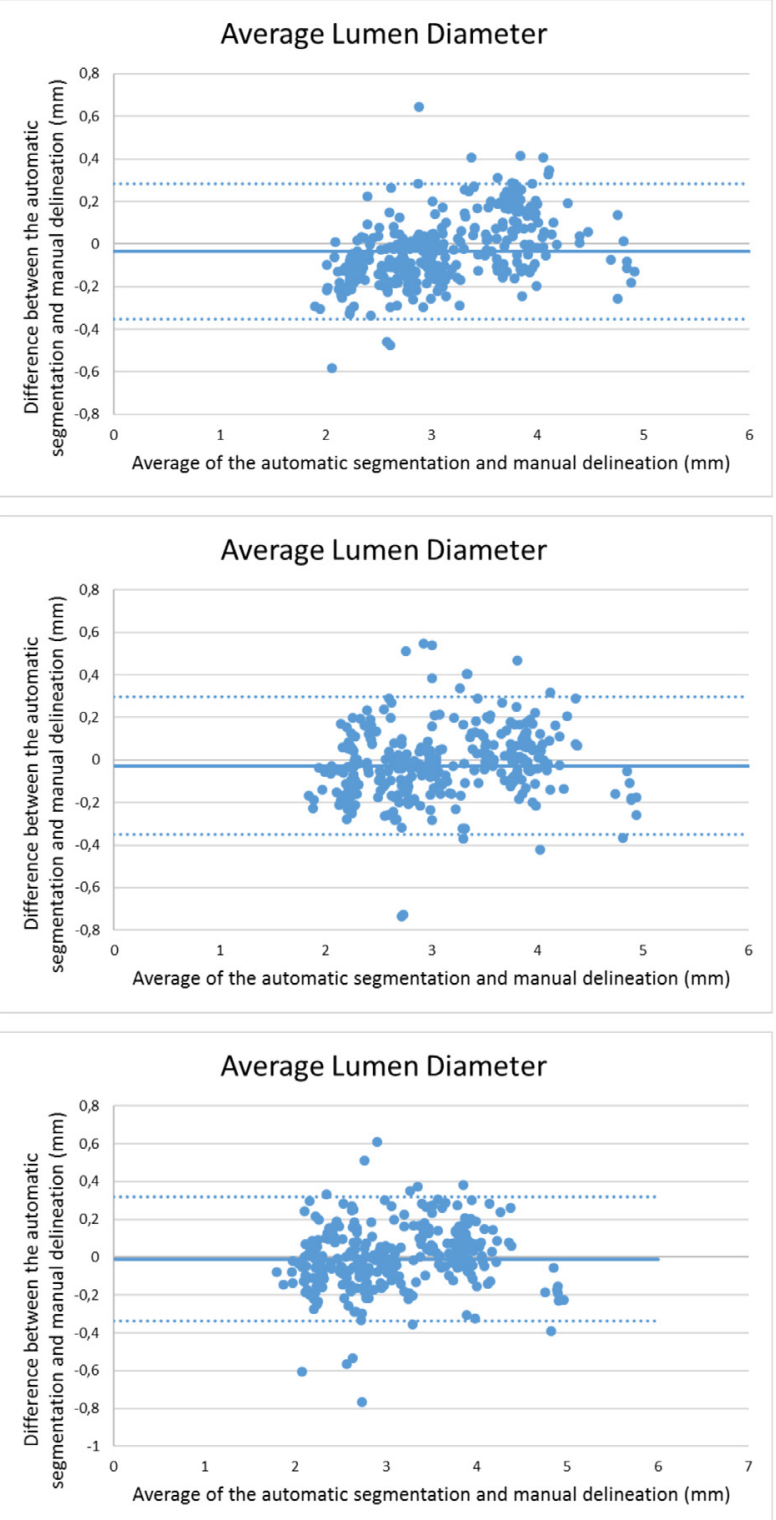

Fig. 12. Bland-Altman plots of the average lumen diameter of the automatically segmented lumen and the three corresponding manual delineations.

delineations reduced when the contrast enhancement was applied. Similar reductions of the bias were also obtained for the average lumen diameter. When compared to the manual delineation 3, a reduction of the bias from $-0.02701 \mathrm{~mm}$ to $-0.00963 \mathrm{~mm}$ was obtained before and after adjusting the contrast, respectively, leading to an average distance less than one pixel between the automatic and manual segmentations.

The high complexity $\left(O\left(d^{2}, N^{2}\right)\right)$ of the subtractive clustering algorithm makes it unfeasible to be applied in images with higher resolutions due to the high number of pixels to be processed to find the centroids of the clusters. Hence, the reduction of the resolution of the input image in order to decrease the computational cost of the subtractive clustering algorithm was tackled in this study. The Gaussian pyramid proved to be the most suitable choice due to its simple implementation and effective reduction of the resolution of the input image without losing important information on the structure of interest. The effectiveness of the Gaussian pyramid can be perceived from the data in Table 8, which indicates that the execution time of the K-means with the subtractive clustering algorithm is low when compared to the average total time and the time required by the Chan-Vese algorithm. This is due to the reduction of the number of pixels to be processed by the subtractive clustering algorithm when the Gaussian pyramid was applied. The computational cost of the Chan-Vese active contour was greater than that of the other algorithms. Since re-initialization of the signed distance function of the contour is necessary at every step of the contour evolution, the time consumed by the algorithm increases when applied to images of higher resolution. However, it was decided to apply the Chan-Vese active contour to the original IVUS image in order to take into account all the pixels of the lumen region available in the full resolution of the input image.

\subsection{Comparison with the intra- and inter-observer variability}

In terms of the inter-observer variability, i.e. the comparison between the manual delineations of Expert 1 and Expert 2, the results are close to the ones computed from our method. The aver- 

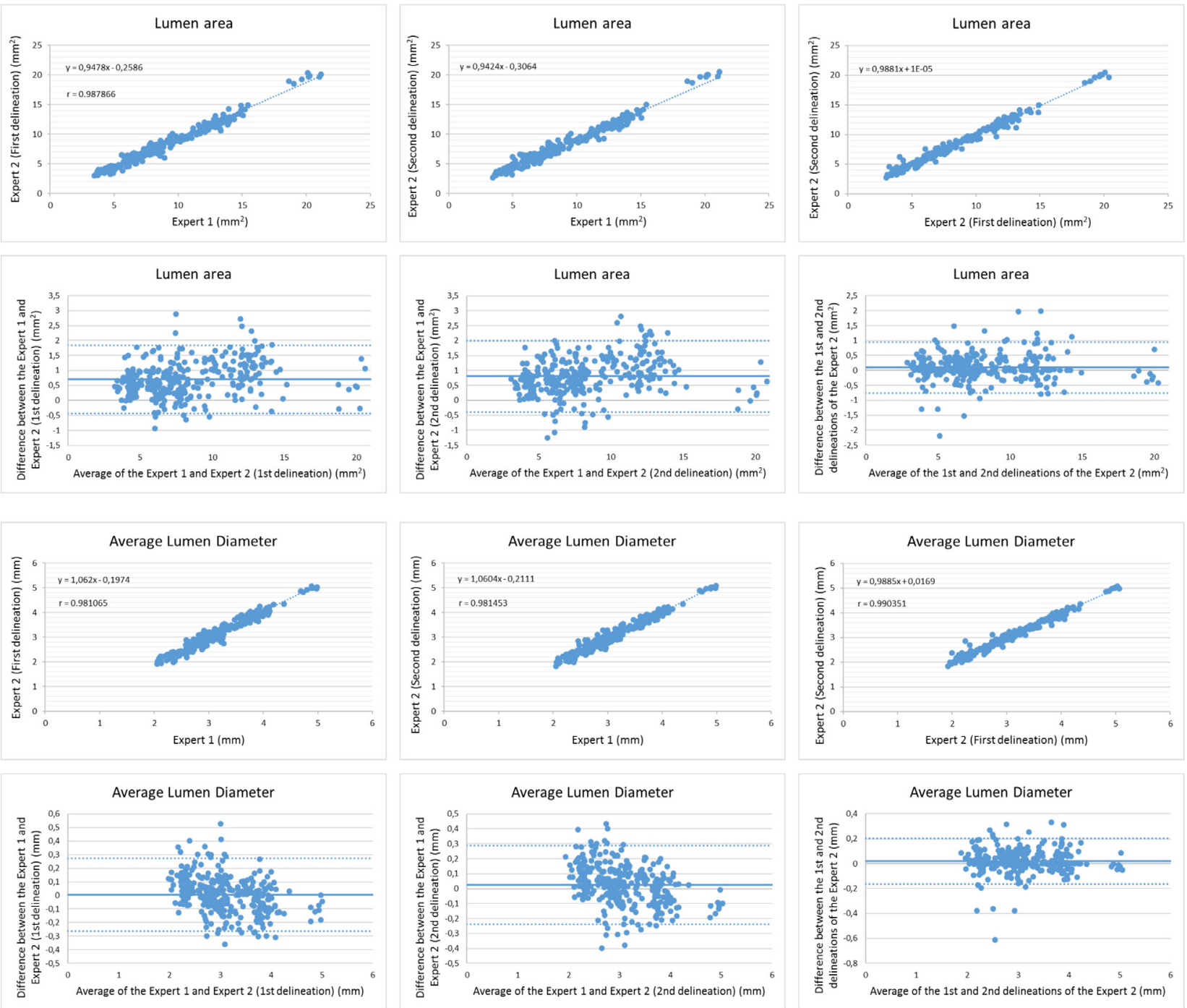

Fig. 13. Linear regression and Bland-Altman analysis concerning the lumen area and average lumen diameter obtained from the delineations of the two experts.

age values of the Jaccard measure, Hausdorff distance, percentage of area difference and Dice coefficient of Expert 1 in comparison to the first delineation of Expert 2 were $0.88 \pm 0.05,0.28 \pm 0.13 \mathrm{~mm}$, $0.11 \pm 0.08$ and $0.94 \pm 0.03$, respectively. The average values for the same measures obtained from the comparison between the proposed method after adjusting the contrast of the input images and the first delineation of Expert 2 (represented by the manual delineation 2 in Table 3 ) were $0.88 \pm 0.06,0.29 \pm 0.17 \mathrm{~mm}, 0.08$ \pm 0.07 and $0.94 \pm 0.04$, respectively. Regarding the comparison between the manual delineation of Expert 1 and the second delineation of Expert 2, the average values of the Jaccard measure, Hausdorff distance, percentage of area difference and Dice coefficient were $0.87 \pm 0.05,0.30 \pm 0.13 \mathrm{~mm}, 0.13 \pm 0.08$ and 0.93 \pm 0.03 , respectively. The results of the proposed method for the same measures obtained from the comparison with the second delineation of Expert 2 (represented by the manual delineation 3 in Table 3) were $0.88 \pm 0.06,0.29 \pm 0.18 \mathrm{~mm}, 0.08 \pm 0.08$ and 0.94 \pm 0.04 , respectively, which were slightly better than the ones obtained between Expert 1 and Expert 2 (second delineation).

The regression analysis showed that the segmentation results of the proposed method are also close to ones obtained from the intra- and inter-observer variability. In terms of the lumen area, the Pearson correlation coefficients between Expert 1 and the first and second delineations of Expert 2 were 0.987866 and 0.986466 , respectively. The regression analysis of the intra-observer variability showed a correlation coefficient of 0.992330 between the lumen areas of the first and second delineations of Expert 2. After the application of the contrast enhancement, the automatic segmentation method obtained a correlation coefficient of 0.985729 , 0.974483 and 0.974314 with the lumen area calculated from the manual delineations of Expert 1 and the first and second delineations of Expert 2, respectively. For the average lumen diameter, the correlation coefficients between Expert 1 and the first and second delineations of Expert 2 were 0.981065 and 0.981453 , respectively, whereas the correlation coefficient between the first and second delineations of Expert 2 was 0.990351. After adjusting the contrast of the input images, the automatic segmentation of the lumen obtained a correlation coefficient of 0.977346, 0.972354 and 0.971756 with the average lumen diameter of the manual delineations of Expert 1 and the first and second delineations of Expert 2 , respectively. The Bland-Altman analysis showed similar results between the proposed method and the intra- and inter-observer variability. For the lumen area, the bias between Expert 1 and the first delineation of Expert 2 were $0.708995 \mathrm{~mm}^{2}$, whereas for the second delineation of Expert 2 the bias was $0.803415 \mathrm{~mm}^{2}$. The Bland-Altman analysis of the lumen area with respect to the intra-observer variability showed a bias of $0.094420 \mathrm{~mm}^{2}$ between the first and second delineations of Expert 2. The bias be- 
tween the proposed method after adjusting the contrast of the images and the first delineation of Expert 2 was $-0.09497 \mathrm{~mm}^{2}$, which is closer to zero when compared to the bias between Expert 1 and the same delineation of Expert $2\left(0.708995 \mathrm{~mm}^{2}\right)$. For the average lumen diameter, the bias between Expert 1 and the first delineation of Expert 2 was $0.006241 \mathrm{~mm}$, whereas for the second delineation of Expert 2 the bias was $0.024817 \mathrm{~mm}$. For the intra-observer variability, the bias of the average lumen diameter between the first and second delineations of Expert 2 was $0.018575 \mathrm{~mm}$. The bias of the average lumen diameter between the proposed method after adjusting the contrast of the images and the three manual delineations were $-0.03283 \mathrm{~mm}$, $0.02680 \mathrm{~mm}$ and $-0.00963 \mathrm{~mm}$. Compared to the manual delineations, the proposed method underestimated the average lumen diameter. However, the results were similar to the intra- and interobserver variability.

\subsection{Comparison with other methods}

A comparison with related studies found in the literature was carried out in order to validate the accuracy of the proposed lumen segmentation method. The first comparison was performed against the results obtained in the IVUS Segmentation Challenge (Balocco et al., 2014). The average values described in Table 6 showed that the method proposed by Participant group 3 outperformed the ones obtained from the other participant groups. A slight improvement of the Hausdorff distance was obtained from our method when compared to the one of Participant group 3 of the challenge $(0.29 \pm 0.17 \mathrm{~mm}$ vs $0.34 \pm 0.14 \mathrm{~mm}$, respectively). Although no significant improvements were achieved when compared to the results of this participant group, the segmentation of the lumen performed by our method is fully automatic and there is no need for an initial user interaction. In contrast, the method proposed by this group requires the initialization of a number of points to generate the initial contours.

The results shown in Table 7 indicate that our method outperformed many of the studies found in the literature. The value of the Dice coefficient of our method $(0.9400 \pm 0.0400)$ is better than the values obtained by Sofian et al. (2015) and Mendizabal-Ruiz et al. (2013) ( $0.9260 \pm 0.0111$ and $0.9283 \pm 0.0201$, respectively). The value of the Jaccard measure $(0.8800 \pm 0.0600)$ was also better than the ones obtained from the studies under comparison, except for the value obtained by Su et al. (2016) (0.9182). The value of the Hausdorff distance $(0.2900 \pm 0.1700 \mathrm{~mm})$ is only greater than the ones obtained from Su et al. (2016) and Mendizabal-Ruiz et al. (2013) (0.2243 $\mathrm{mm}$ and $0.1398 \pm 0.0384 \mathrm{~mm}$, respectively). Finally, the value of the percentage of area difference $(0.0900 \pm$ 0.0700 ) is only greater than the one obtained from Sofian et al. (2015) (0.0645 \pm 0.0509$)$.

Although the results presented by Su et al. (2016) are better than the ones obtained from our method, a ROI surrounding the region corresponding to the media-adventitia region must be delineated in the input image before the classification by the two ANN. In addition, the elimination of noise of the binary image resulting from the first classification is performed by a second ANN. In contrast, morphological operations and the convex hull algorithm are used here to identify noise and refine the regions to be evaluated instead of using a complex ANN. In addition, the method proposed performs the lumen segmentation in the whole image. Although the value of the percentage of area difference of the method proposed by Sofian et al. (2015) is less than the one obtained from the proposed method, the authors only used 30 IVUS images randomly selected from the dataset provided by Balocco et al. (2014) to perform the experiments. In the study tackled by Mendizabal-Ruiz et al. (2013), IVUS images acquired from catheters operating at frequencies of $20 \mathrm{MHz}$ and $40 \mathrm{MHz}$ were taken into account. However, if only the images acquired from the frequency of $20 \mathrm{MHz}$ were considered, then the average values of the Dice, Jaccard and Hausdorff distance would be $0.9215 \pm 0.0217,0.8555$ \pm 0.0363 and $0.1421 \pm 0.0371 \mathrm{~mm}$, respectively.

In terms of the computational cost, the average total time of the proposed method is also in accordance with the related studies found in the literature. The average total time of the proposed method to process each one of the 326 IVUS images was $5.7165 \pm$ 1.5419 s. Gao et al. (2015) reported an average total time of $8.13 \pm$ $6.05 \mathrm{~s}$ for the lumen segmentation step of their automated framework. The average total time of the lumen segmentation carried out by Mendizabal-Ruiz et al. (2013) was $4.51 \mathrm{~s}$.

\subsection{Limitations}

The proposed method has two limitations. The first limitation regards the number of parameters of the clustering algorithm. The K-means algorithm with subtractive clustering used requires three parameters: the number of clusters and the radius $r_{a}$ and $r_{b}$ representing the neighbourhood of the pixels under analysis. Although the proposed method with the same parameters adopted in our previous study for MR images was able to identify the lumen region in IVUS images, a fully automatic segmentation without any empirical parameters increases the reliability and robustness of the method. Hence, future studies will be conducted to effectively separate the regions present in the input images without any kind of parameter.

The second limitation regards the segmentation of IVUS images acquired from a frequency of $40 \mathrm{MHz}$. Since speckle noise in these images is higher when compared to IVUS images acquired at a frequency of $20 \mathrm{MHz}$, the application of most efficient filters to minimize the effects of such noisy artefacts will be considered in future research projects to enable segmentation on images acquired from catheters operating at different frequencies. In addition, texture analysis will also be considered instead of using only the grayscale intensity of the pixels in the K-means algorithm.

\section{Conclusions}

The segmentation of the lumen and media-adventitia regions in IVUS images is an intensive focus of research and plays an important role in assessing the presence and progression of atherosclerosis. A fully automatic segmentation of the lumen in IVUS images of coronary arteries was proposed in this article. Compared to our previous study, new solutions were developed to enhance the robustness, efficiency and automaticity of the proposed method. Additionally, a new approach to successfully identify and remove side branches of bifurcation regions was also proposed to avoid the elimination of the potential lumen regions from the subsequent processing steps. The improved method proved to be effective in identifying the regions corresponding to the lumen without user interaction and any change in the values of the method parameters.

Modifications were also accomplished to improve the shape of the lumen contour and the segmentation accuracy. The qualitative analysis showed that the visual shape of the lumen contour produced by the new contour correction step was better than the one obtained from the Chan-Vese active contour algorithm, leading to smoother and more regular final contours. The quantitative analysis demonstrated that the segmentation results of the new automatic segmentation method are in accordance with the manual delineations performed by two experts. Additionally, the proposed method showed results close to the ones obtained from the interobserver variability.

An effective approach to reduce the number of parameters of the subtractive clustering algorithm, as well as the application of 
the method proposed here to IVUS images acquired from catheters operating at different frequencies, are expected to be addressed in future research work.

\section{Acknowledgements}

This work was partially funded by Coordenação de Aperfeiçoamento de Pessoal de Nível Superior (CAPES), funding agency in Brazil, under the PhD Grant with reference number 0543/13-6.

The authors thank the funding of Project NORTE-01-0145FEDER-000022 - SciTech - Science and Technology for Competitive and Sustainable Industries, co-financed by "Programa Operacional Regional do Norte" (NORTE2020), through "Fundo Europeu de Desenvolvimento Regional" (FEDER).

\section{References}

Adelson, E.H., Anderson, C.H., Bergen, J.R., Burt, P.J., Ogden, J.M., 1984. Pyramid methods in image processing. RCA Engineer 29 (6), 33-41.

Balocco, S., Gatta, C., Ciompi, F., Wahle, A., Radeva, P., Carlier, S., Unal, G., Sanidas, E., Mauri, J., Carillo, X., Kovarnik, T., Wang, C.-W., Chen, H.-C., Exarchos, T.P., Fotiadis, D.I., Destrempes, F., Cloutier, G., Pujol, O., Alberti, M., MendizabalRuiz, E.G., Rivera, M., Aksoy, T., Downe, R.W., Kakadiaris, I.A., 2014. Standardized evaluation methodology and reference database for evaluating IVUS image segmentation. Comput. Med. Imaging Graph. 38 (2), 70-90. Special Issue on Computing and Visualisation for Intravascular Imaging. http://dx.doi.org/10. 1016/j.compmedimag.2013.07.001

Bataineh, K., Naji, M., Saqer, M., 2011. A comparison study between various fuzzy clustering algorithms. Editorial Board 5 (4), 335-343.

Berg, M.d., Cheong, O., Kreveld, M.v., Overmars, M., 2008. Computational geometry: Algorithms and applications, third ed. Springer-Verlag TELOS, Santa Clara, CA, USA.

Broersen, A., de Graaf, M.A., Eggermont, J., Wolterbeek, R., Kitslaar, P.H., Dijkstra, J., Bax, J.J., Reiber, J.H.C., Scholte, A.J., 2016. Enhanced characterization of calcified areas in intravascular ultrasound virtual histology images by quantification of the acoustic shadow: validation against computed tomography coronary angiography. Int. J. Cardiovasc. Imaging 32 (4), 543-552. doi:10.1007/ s10554-015-0820-x.

Cardinal, M.H.R., Meunier, J., Soulez, G., Maurice, R.L., Therasse, E., Cloutier, G., 2006. Intravascular ultrasound image segmentation: a three-dimensional fastmarching method based on gray level distributions. IEEE Trans. Med. Imaging 25 (5), 590-601. doi:10.1109/TMI.2006.872142.

Cardinal, M.-H.R., Soulez, G., Tardif, J.-C., Meunier, J., Cloutier, G., 2010. Fast-marching segmentation of three-dimensional intravascular ultrasound images: a preand post-intervention study. Med. Phys. 37 (7), 3633-3647.

Chan, T.F., Vese, L.A., 2001. Active contours without edges. IEEE Trans. Image Process. 10 (2), 266-277. doi:10.1109/83.902291.

Chiu, S.L., 1994. Fuzzy model identification based on cluster estimation. J. Intell. Fuzzy Syst. 2 (3), 267-278.

Destrempes, F., Roy Cardinal, M.-H., Allard, L., Tardif, J.-C., Cloutier, G., 2014. Segmentation method of intravascular ultrasound images of human coronary arteries. Comput. Med. Imaging Graph. 38 (2), 91-103. doi:10.1016/j.compmedimag. 2013.09.004.

Dhanachandra, N., Manglem, K., Chanu, Y.J., 2015. Image segmentation using Kmeans clustering algorithm and subtractive clustering algorithm. Procedia Comput. Sci. 54, 764-771. doi:10.1016/j.procs.2015.06.090.

Diethrich, E.B., Irshad, K., Reid, D.B., 2006. Virtual histology and color flow intravascular ultrasound in peripheral interventions. In: Seminars in Vascular Surgery, 19. Elsevier, pp. 155-162.

Gao, Z., Hau, W.K., Lu, M., Huang, W., Zhang, H., Wu, W., Liu, X., Zhang, Y.-T., 2015. Automated framework for detecting lumen and media-adventitia borders in intravascular ultrasound images. Ultrasound Med. Biol. 41 (7), 2001-2021. doi:10.1016/j.ultrasmedbio.2015.03.022.

Huang, C., Zeng, L., 2015. An active contour model for the segmentation of images with intensity inhomogeneities and bias field estimation. PLOS ONE 10 (4), 124. doi:10.1371/journal.pone.0120399.
Huang, S.-C., Cheng, F.-C., Chiu, Y.-S., 2013. Efficient contrast enhancement using adaptive gamma correction with weighting distribution. IEEE Trans. Image Process. 22 (3), 1032-1041. doi:10.1109/TIP.2012.2226047.

Jain, S., Jagtap, V., Pise, N., 2015. Computer aided melanoma skin cancer detection using image processing. Procedia Comput. Sci. 48, 735-740. doi:10.1016/j.procs. 2015.04.209.

Jodas, D.S., Pereira, A.S., Tavares, J.M.R., 2016. Lumen segmentation in magnetic resonance images of the carotid artery. Comput. Biol. Med. 79, 233-242. http: //dx.doi.org/10.1016/j.compbiomed.2016.10.021.

Kass, M., Witkin, A., Terzopoulos, D., 1988. Snakes: active contour models. Int. J. Comput. Vis. 1 (4), 321-331. doi:10.1007/BF00133570

Katouzian, A., Angelini, E.D., Carlier, S.G., Suri, J.S., Navab, N., Laine, A.F., 2012. A state-of-the-art review on segmentation algorithms in intravascular ultrasound (IVUS) images. IEEE Trans. Inf. Technol. Biomed. 16 (5), 823-834. doi:10.1109/ TITB.2012.2189408

König, A., Klauss, V., 2007. Virtual histology. Heart 93 (8), 977-982. doi:10.1136/hrt. 2007.116384.

Lo Vercio, L., Orlando, J.I., del Fresno, M., Larrabide, I., 2016. Assessment of image features for vessel wall segmentation in intravascular ultrasound images. Int. J. Comput. Assist. Radiol. Surg. 11 (8), 1397-1407. doi:10.1007/s11548-015-1345-4.

Ma, Z., Tavares, J.M.R.S., Jorge, R.N., Mascarenhas, T., 2010. A review of algorithms for medical image segmentation and their applications to the female pelvic cavity. Comput Methods Biomech Biomed Engin 13 (2), 235-246. doi:10.1080/ 10255840903131878

de Macedo, M.M.G., Takimura, C.K., Lemos, P.A., Gutierrez, M.A., 2016. A robust fully automatic lumen segmentation method for in vivo intracoronary optical coherence tomography. Res. Biomed. Eng. 32 (1), 35-43. doi:10.1590/2446-4740.0759.

Mendis, S., Puska, P., Norrving, B., 2011. Global Atlas on Cardiovascular Disease Prevention and Control. World Health Organization.

Mendizabal-Ruiz, E.G., Rivera, M., Kakadiaris, I.A., 2013. Segmentation of the luminal border in intravascular ultrasound b-mode images using a probabilistic approach. Med. Image Anal. 17 (6), 649-670. http://dx.doi.org/10.1016/j.media. 2013.02.003.

Moraes, M.C., Furuie, S.S., 2011. Automatic coronary wall segmentation in intravascular ultrasound images using binary morphological reconstruction. Ultrasound Med. Biol. 37 (9), 1486-1499. doi:10.1016/j.ultrasmedbio.2011.05.018.

Nair, A., Kuban, B.D., Tuzcu, E.M., Schoenhagen, P., Nissen, S.E., Vince, D.G., 2002. Coronary plaque classification with intravascular ultrasound radiofrequency data analysis. Circulation 106 (17), 2200-2206.

Ritter, N., Cooper, J., 2009. New resolution independent measures of circularity. J. Math. Imaging Vis. 35 (2), 117-127. doi:10.1007/s10851-009-0158-X.

Santos, A.M.F., dos Santos, R.M., Castro, P.M.A., Azevedo, E., Sousa, L., Tavares, J.M.R.S., 2013. A novel automatic algorithm for the segmentation of the lumen of the carotid artery in ultrasound B-mode images. Expert Syst. Appl. 40 (16), 6570-6579. doi:10.1016/j.eswa.2013.06.003.

dos Santos, E., Yoshizawa, M., Tanaka, A., Saijo, Y., Iwamoto, T., 2005. Detection of luminal contour using fuzzy clustering and mathematical morphology in intravascular ultrasound images. In: IEEE 27th Annual Conference Engineering in Medicine and Biology, pp. 3471-3474. doi:10.1109/IEMBS.2005.1617226.

Sofian, H., Ming, J.T.C., Noor, N.M., 2015. Detection of the lumen boundary in the coronary artery disease. In: 2015 IEEE International WIE Conference on Electrical and Computer Engineering (WIECON-ECE). IEEE, pp. 143-146. doi:10.1109/ WIECON-ECE.2015.7443882.

Su, S., Hu, Z., Lin, Q., Hau, W.K., Gao, Z., Zhang, H., 2017. An artificial neural network method for lumen and media-adventitia border detection in IVUS. Comput. Med. Imaging Graph. 57, 29-39. doi:10.1016/j.compmedimag 2016.11.003.

Sun, Z.-H., Wei, X.-H., Zhou, W.-H., 2012. A nyström-based subtractive clustering method. In: 2012 International Conference on Wavelet Active Media Technology and Information Processing (ICWAMTIP), pp. 74-77. doi:10.1109/ICWAMTIP. 2012.6413443

Taki, A., Najafi, Z., Roodaki, A., Setarehdan, S.K., Zoroofi, R.A., Konig, A., Navab, N., 2008. Automatic segmentation of calcified plaques and vessel borders in IVUS images. Int. J. Comput. Assist. Radiol. Surg. 3 (3), 347-354. doi:10.1007/ s11548-008-0235-4.

Vard, A., Jamshidi, K., Movahhedinia, N., 2012. An automated approach for segmentation of intravascular ultrasound images based on parametric active contour models. Australas. Phys. Eng. Sci. Med. 35 (2), 135-150. doi:10.1007| s13246-012-0131-7.

Wang, X.-F., Huang, D.-S., Xu, H., 2010. An efficient local Chan-Vese model for image segmentation. Pattern Recognit. 43 (3), 603-618. http://dx.doi.org/10.1016/j. patcog.2009.08.002. 